TRANSACTIONS OF THE

AMERICAN MATHEMATICAL SOCIETY

Volume 354, Number 2, Pages 537-569

S 0002-9947(01)02878-1

Article electronically published on September 21, 2001

\title{
ON THE STRUCTURE OF SPECTRA OF PERIODIC ELLIPTIC OPERATORS
}

\author{
PETER KUCHMENT AND SERGEI LEVENDORSKIÎ
}

\begin{abstract}
The paper discusses the problem of absolute continuity of spectra of periodic elliptic operators. A new result on absolute continuity for a matrix operator of Schrödinger type is obtained. It is shown that all types of operators for which the absolute continuity has previously been established can be reduced to this one. It is also discovered that some natural generalizations stumble upon an obstacle in the form of non-triviality of a certain analytic bundle on the two-dimensional torus.
\end{abstract}

\section{INTRODUCTION}

Elliptic differential operators with periodic coefficients arise naturally in many areas of mathematical physics. One can mention among the most prominent examples quantum solid state theory, where the main operator of interest is the stationary Schrödinger operator

$$
-\Delta+V(x)
$$

with a potential $V(x)$ periodic with respect to a lattice in $\mathbb{R}^{d}$ (see for instance [4, 54]).

Another example that has gained importance due to recent advances in the theory of photonic crystals (e.g., 32, 38]) is the periodic Maxwell operator, one of the realizations of which (in fact, of its square) can be written as

$$
\frac{1}{\mu(x)} \nabla \times \frac{1}{\varepsilon(x)} \nabla \times
$$

defined on zero-divergence vector fields in $\mathbb{R}^{3}$. Here the periodic functions $\varepsilon(x)$ and $\mu(x)$ represent the electric permittivity and magnetic permeability of the medium.

Scalar counterparts of this operator are

$$
-\nabla \cdot \frac{1}{\varepsilon(x)} \nabla
$$

and

$$
-\frac{1}{\mu(x)} \Delta
$$

Received by the editors October 3, 2000.

2000 Mathematics Subject Classification. Primary 35P99; Secondary 35J10.

Key words and phrases. Periodic operator, elliptic operator, absolutely continuous spectrum, Schrödinger operator, magnetic and electric potentials.

The first author was supported in part by an NRC COBASE Grant, NSF Grants DMS 9610444 and DMS 0072248, and by a DEPSCoR Grant.

The second author was supported in part by an NRC COBASE Grant. 
(in the latter case the operator must be considered in a weighted $L_{2}$-space in order to be self-adjoint). These scalar operators also arise in studying periodic acoustic media.

Among other operators of interest one can mention the divergence type operators

$$
\sum \partial_{i} a_{i j}(x) \partial_{j}
$$

and magnetic Schrödinger operators

$$
\left(\frac{1}{i} \nabla-A(x)\right)^{2}+V(x),
$$

where the scalar functions $a_{i j}(x)$ and $V(x)$ and the vector magnetic potential $A(x)$ are assumed to be periodic with respect to a lattice.

Another extensively studied case is that of a periodic Dirac operator

$$
\frac{1}{i} \sum_{j=1}^{d} \frac{\partial}{\partial x_{j}} \alpha_{j}+V(x),
$$

where $\alpha_{j}$ are self-adjoint matrices satisfying the anti-commutation relations

$$
\alpha_{j} \alpha_{i}+\alpha_{i} \alpha_{j}=2 \delta_{i j} I
$$

(Dirac or Pauli matrices) and the periodic matrix function $V$ is the potential.

In all these cases the structure of the spectrum of the corresponding operator is of major interest. Consider for instance the periodic Schrödinger operator (1) in $\mathbb{R}^{d}$. Assuming that the potential is real and satisfies some mild conditions, one can prove self-adjointness of this operator (see [54]). It is well known that the spectrum of such an operator has the so-called band-gap structure, i.e. it consists of the union of finite closed segments $\left[a_{j}, b_{j}\right]$ such that $a_{j} \rightarrow \infty$ (e.g. [4], 28], [32], 34, 37], [54, [61]). It has been known to physicists for a long time (see for instance [4]) that the spectrum of this operator in $L_{2}\left(\mathbb{R}^{d}\right)$ does not contain any eigenvalues. Put in different terms, this means that none of the spectral bands $\left[a_{j}, b_{j}\right]$ collapses into a point. One can easily prove that there are no eigenvalues of finite multiplicity 28]; but the proof of the general statement had to wait for a long time, until the celebrated Thomas' theorem was proven in 1973 [68]. In fact, the paper [68] contained the proof of a more general statement: the spectrum of a self-adjoint periodic operator (11) in $3 D$ is absolutely continuous. One should notice that the statement on absence of eigenvalues holds also in the non-self-adjoint case. In the self-adjoint situation the so-called Floquet theory makes it easy to deduce absolute continuity from the absence of eigenvalues, since one can easily prove absence of the singular continuous spectrum (see for instance [37, [54, and [60]). Although the proofs of this implication in [37, [54], and 60] are formulated for the Schrödinger case only, the derivation is very general and holds for any self-adjoint periodic elliptic operator with sufficiently regular coefficients (see for instance the proof of Theorem 4.5.9 in [37). In fact, the statement on absence of the singular continuous spectrum holds with the same proof for operators in periodic electro-magnetic fields in the case of a non-zero rational magnetic flux through the unit cell (i.e., when the magnetic potential is not periodic). Then one has an abelian group of the so-called "magnetic translations" (or Zak group, e.g. [26], [27], [50], and [71]), which enables one to carry through the proof. In this situation, however, a point spectrum can arise (Landau levels). 
The question arises on whether absence of eigenvalues (absolute continuity of spectrum in the self-adjoint case) is a property that all periodic elliptic differential operators enjoy. It is known, however (see pp. 135-136 in [37]) that this is in general not true for elliptic operators of order four. On the other hand, the common belief is that periodic elliptic operators of second order do not have eigenvalues (or, in the self-adjoint case, have absolutely continuous spectra), although it is rarely discussed why this must be true. Let us describe why the authors believe in this. Assuming that a periodic elliptic operator has an eigenvalue, one can derive from this the existence of an eigenfunction of a super-exponential decay (see Theorems 4.1.5 and 4.1.6 in [37] and Theorem 12 in [36]). Existence of such fast decaying solutions is possible for fourth order equations, where even compactly supported solutions can exist (see pp. 135-136 in [37 and the original paper [53]). In the case of equations of second order, such fast decay should probably violate uniqueness of continuation at infinity and hence be impossible. Unfortunately, the appropriate uniqueness theorems that prohibit super fast decay at infinity for equations of the type we consider are not known yet in full generality. The only applicable results of [30] and [46] deal with the Schrödinger operator with an electric potential only. Thus, due to the absence of appropriate theorems of possible decay rate of solutions, the absolute continuity problem since Thomas' paper [68 has been approached in a different way. We recall this in Section 2. Let us list the known results. Thomas' theorem [68] was extended to all dimensions and a broader class of periodic Schrödinger operators in [54. Papers [16] 20] contained the proof of the absolute continuity of the spectrum for the Dirac operator with a periodic scalar electric potential. Thomas' result for the Schrödinger operator implies the similar statement for the operator

$$
H_{\rho, V}=-\nabla \cdot \rho^{2}(x) \nabla+V(x)
$$

with periodic functions $\rho \neq 0$ and $V$ if $\rho$ is smooth enough, due to the well known transformation

$$
\rho^{-1} H_{\rho, V} \rho^{-1},
$$

which reduces the operator to the form (1). The disadvantage of this transform is that it requires smoothness of $\rho$, a condition which is probably not needed for the result to hold and which is violated in many applications (for instance, to photonic crystals). Thomas' theorem also handles the operator (4). However, the case of the magnetic Schrödinger operator (6) had resisted attempts to prove absolute continuity of the spectrum until the paper [31, where this was done for the case of small magnetic potentials. Finally, in the remarkable papers [8] and 65] the full-strength statement about the magnetic Schrödinger operator was proven first in dimension two 8 and then in arbitrary dimension [65]. The elegant algebraic approach of [8] works only in dimension two, but it nevertheless led to a proof in arbitrary dimension [65]. Papers [8] and 65] triggered an avalanche of publications that significantly advanced the known results (see [9]-[14], 21]-[24], [41], [47], [48, 55]-[58], 66], and 63]). Some major questions, however (for instance, how to handle operators with variable coefficients in the higher order terms) remain unanswered. One can find a rather comprehensive survey in [12] and 63].

The goal of this paper is to provide a new absolute continuity result for a matrix Schrödinger operator such that all operators treated so far can be transformed into this form. The proof uses microlocal analysis techniques and employs an idea from [65] in a simplified form. We were also influenced by the papers [49] and 
63], although this might not be obvious from the text. We believe that besides obtaining new results, this approach clarifies and unifies the known ones. At the same time it singles out a problem of triviality of a certain analytic vector bundle on the torus, an obstruction which has not been noticed before. We would also like to mention that our main results do not require self-adjointness. This happens to be useful even for some self-adjoint problems, which can sometimes be transformed to simpler non-self-adjoint ones, as it is explained in Section 7 A limited version of the main result was announced in [41].

We want to make it clear that we did not even attempt to achieve optimal conditions on the coefficients of the equations, but rather to encompass a wider set of types of equations. Finding the best possible conditions on the coefficients is an important task (especially taking into account that in many applications, for instance to photonic crystals 32, 38, the coefficients in the leading terms are piecewise constant). This was, however, not among our goals, and so our conditions on coefficients are in most cases not optimal. One can find optimal or close to optimal conditions for some cases in the recent papers [12, [55], 56].

Let us describe the structure of the paper. The next section provides a preliminary discussion of the problem, in particular an exposition of Thomas' original approach [68. Section 3 contains the formulation of the main result of the paper. The next section was written for convenience of the reader and provides a brief sketch of the proof. Section 5 delivers the proof of the main result in the absence of electric potential. In the next section, the known technique of including the electrical potential is indicated with appropriate references. Section 7 describes the algebraic reduction of all types of operators studied so far to the form contained in our main result. The paper ends with concluding remarks and acknowledgments.

\section{Formulation of the PRoblem AND PRELIMinary Discussion}

Let $P(x, D)$ be an elliptic second order scalar or matrix differential operator in $\mathbb{R}^{d}$ with coefficients periodic with respect to a lattice $\Gamma$ in $\mathbb{R}^{d}$. Here $D=-i \nabla$. For simplicity of presentation we will consider mostly the case of the integer lattice $\mathbb{Z}^{d}$. It is easy, however, to modify the proofs to cover an arbitrary lattice. We will not yet specify any conditions on smoothness of the coefficients of $P$. For now the reader can assume the coefficients to be as smooth as needed. The natural domain of definition of the operator will then be the Sobolev space $H^{2}\left(\mathbb{R}^{d}\right)$ (or its vector analog in the case of systems, although we will not distinguish these cases in the notations for the spaces). Our goal is to prove nonexistence of non-zero $L_{2^{-}}$ solutions of the equation $P u=\lambda u$. As it was mentioned in the introduction, in the self-adjoint case standard considerations show that this implies absolute continuity of the spectrum of $P$ in $L_{2}\left(\mathbb{R}^{d}\right)$. We also note that the spectral parameter $\lambda$ can be included in the operator, and hence we will assume from now on that $\lambda=0$.

We will now describe the approach of [68] to the proof of absolute continuity of the spectrum. The reader should be aware that we will adhere to the language of multi-dimensional analyticity, although the original paper [68] and most other publications on the subject use one-dimensional directional analyticity. The language of several complex variables is not only natural, but often unavoidable in the theory of periodic PDEs, as one can conclude for instance from [35], [36], 42] [43], [44], and [52]. This is in parallel with the theory of PDEs with constant coefficients,

where complex analysis in several variables is often the language of choice (see for 
instance [29] and [51]). Using the technique of analytic Fredholm operator functions in several complex variables (see [70], [37]) makes the methods more flexible, allowing for a wide class of equations, including non-self-adjoint ones.

The main technique of Floquet theory (see e.g. [28], [36], [37], [54], [59], [60], and 61]) is the decomposition of the operator $P$ in the space $L_{2}\left(\mathbb{R}^{d}\right)$ into the direct integral of operators on the torus $\mathbb{T}^{d}=\mathbb{R}^{d} / \mathbb{Z}^{d}$ :

$$
P=\int_{B}^{\oplus} P(k) d k
$$

where

$$
B=\left\{k \in \mathbb{R}^{d} \mid k_{j} \in[-\pi, \pi], j=1, \ldots, d\right\}
$$

is the so-called Brillouin zone, and the operator

$$
P(k)=P(x, D+k)
$$

acts on $\mathbb{Z}^{d}$-periodic functions, i.e. on functions on the torus

$$
\mathbb{T}^{d}=\mathbb{R}^{d} / \mathbb{Z}^{d}
$$

The vector $k$ is usually called quasimomentum. This direct integral decomposition provides an isometry between $L_{2}\left(\mathbb{R}^{d}\right)$ and

$$
\int_{B}^{\oplus} L_{2}\left(\mathbb{T}^{d}\right) d k
$$

Let us observe now that one can define the operator family $P(k)$ for all values $k \in \mathbb{C}^{d}$. Then $P(k)$ becomes an analytic (in fact, polynomial) function on $\mathbb{C}^{d}$ with values in the space of bounded Fredholm operators acting from $H^{2}\left(\mathbb{T}^{d}\right)$ into $L_{2}\left(\mathbb{T}^{d}\right)$. Consider now the following set, which we will call the Fermi surface:

$$
F=\left\{k \in \mathbb{C}^{d} \mid \exists u \neq 0, P(k) u=0\right\} \subset \mathbb{C}^{d} .
$$

It follows from the general theory of Fredholm operator functions (see for instance [37], 70] and references therein) that the Fermi surface is an analytic set in $\mathbb{C}^{d}$ (see details and references in 35 and 37]). In fact, it is the set of all zeros of an entire function $f(k)$ of a finite order in $\mathbb{C}^{d}$. Assume now that there exists a non-zero solution $u \in L_{2}\left(\mathbb{R}^{d}\right)$ of the equation $P u=0$. After expanding into the direct integral, we conclude immediately that the set of quasimomenta $k \in B$ for which the operator $P(k)$ has a non-trivial kernel must have positive measure. On the other hand, this set is the intersection $F \cap B$. We come to the conclusion that the function $f(k)$ is equal to zero on a set of a positive measure in the real subspace $\mathbb{R}^{d} \subset \mathbb{C}^{d}$. Due to standard uniqueness theorems for analytic functions, this implies that $f(k)$ is identically equal to zero, and hence $F=\mathbb{C}^{d}$. Looking at the definition of the Fermi surface, we see that this leads to the existence of a nontrivial solution of the equation

$$
P(k) u=0
$$

on $\mathbb{T}^{d}$ for arbitrary $k \in \mathbb{C}^{d}$. So, we come to the following conclusion:

Theorem 1 (see Theorem 4.1.5 in 37). If there exists a vector $k \in \mathbb{C}^{d}$ such that the elliptic operator $P(k)$ on torus $\mathbb{T}^{d}$ has zero kernel, then the equation $P u=0$ in $\mathbb{R}^{d}$ has no non-trivial $L_{2}$-solutions. 
Remark 2. 1. For the case of a periodic Schrödinger operator this statement (in a slightly different form) was established by L. Thomas [68]. In the current form, and even for hypoelliptic operators, it is proven in [36], 37].

2. If in the self-adjoint case one applies this theorem to the operators $P-\lambda$ with arbitrary real $\lambda$, one concludes the absolute continuity of the spectrum of the operator $P$ in $\mathbb{R}^{d}$ (e.g., Theorem 4.5.9 in [37]).

Now the task, according to Theorem 1 becomes to find a quasimomentum $k \in$ $\mathbb{C}^{d}$ such that the operator $P(k)$ on the torus $\mathbb{T}^{d}$ is invertible. We will remind the standard procedure of choosing an appropriate vector $k$ in the case of the periodic Schrödinger operator (11) (see [12], [37, [54], [68]). It can be chosen as $k=(\pi+i \rho, 0, \ldots, 0)$ with a large real $\rho$. Indeed, then the operator $P(k)$ is

$$
(D+k)^{2}+V(x) .
$$

Assume for simplicity that $V \in L_{\infty}\left(\mathbb{R}^{d}\right)$ (this assumption can be significantly weakened, as shown for instance in [6], [12, [36, [54, [55], [56, [68). We will show that

$$
\left\|(D+k)^{2} u\right\|_{L_{2}\left(\mathbb{T}^{d}\right)} \geq \pi \rho\|u\|_{L_{2}\left(\mathbb{T}^{d}\right)}
$$

for any $u \in H^{2}\left(\mathbb{T}^{d}\right)$. In order to derive the inequality (9), we use the Fourier expansion

$$
u(x)=\sum_{m \in \mathbb{Z}^{d}} \widehat{u}_{m} e^{2 \pi i m \cdot x} .
$$

Then

$$
(D+k)^{2} u=\sum_{m \in \mathbb{Z}^{d}}(2 \pi m+k)^{2} \widehat{u}_{m} e^{2 \pi i m \cdot x} .
$$

Due to the choice of $k$, we get

$$
\left|(2 \pi m+k)^{2}\right| \geq\left|\operatorname{Im}(2 \pi m+k)^{2}\right|=\left|\left(2 \pi m_{1}+\pi\right) \rho\right| \geq \pi|\rho|,
$$

which proves (9). Now, choosing $\rho$ such that $\pi \rho>\|V\|_{L_{\infty}}$, one concludes that the equality

$$
(D+k)^{2} u+V(x) u=0
$$

is impossible for $u \neq 0$. Then according to Theorem 1 we obtain that the equation

$$
-\Delta u+V u=0
$$

in $\mathbb{R}^{d}$ has no non-zero $L_{2}$-solutions. This proves the following result:

Theorem 3 ([68]). The periodic Schrödinger operator $H$ in $\mathbb{R}^{d}$ has no eigenvalues, and hence in the self-adjoint case its spectrum is absolutely continuous.

Remark 4. The statement on absence of eigenvalues does not require self-adjointness, so it holds even in the case of a complex periodic potential.

\section{The MAIn ReSUlt}

The main object of study in this paper is the following matrix generalization of a periodic magnetic Schrödinger operator (6) in $\mathbb{R}^{d}, d \geq 2$ :

$$
H(G, \mathbf{a}, V)=-\sum_{j, k=1}^{d} \frac{\partial}{\partial x_{j}} g_{j k} \frac{\partial}{\partial x_{k}}-2 i \sum_{j=1}^{d} a_{j}(x) \frac{\partial}{\partial x_{j}}+V(x),
$$


where $G=\left\{g_{j k}\right\}$ is a constant positive definite $d \times d$ matrix, the $n \times n$ matrix function $V(x)$ is the "electric potential" (in fact, it corresponds to a combination of the magnetic and electric potentials in ([6] $)$ ) and the $n \times n$ matrix functions $a_{j}(x)$ form an analog $\mathbf{a}=\left\{a_{j}(x)\right\}$ of a magnetic potential. Both potentials $\mathbf{a}$ and $V$ are assumed to be periodic with respect to a lattice $\Gamma$ in $\mathbb{R}^{d}$. A linear change of variables transforms $\Gamma$ into the integer lattice $\mathbb{Z}^{d}$ without changing the form of the operator, so we will assume from now on that $\Gamma=\mathbb{Z}^{d}$. Although the matrix $G$ cannot be diagonalized without destroying the equality $\Gamma=\mathbb{Z}^{d}$, the proofs will be provided for simplicity for the case when $G=I$ only, and then can be rather automatically adjusted to arbitrary positive definite $G$. So, we will concentrate for the sake of simplicity on the operator

$$
H(\mathbf{a}, V)=-\Delta-2 i \sum_{j=1}^{d} a_{j}(x) \frac{\partial}{\partial x_{j}}+V(x)
$$

with $\mathbb{Z}^{d}$-periodic potentials only, while the results will hold for any operator (10) with an arbitrary lattice of periods.

In order to simplify the notation, we will use standard function spaces notation like $H^{s}\left(\mathbb{T}^{d}\right)$ or $L_{2}\left(\mathbb{T}^{d}\right)$ for vector and matrix valued functions, meaning that each of their entries belongs to the corresponding space.

According to Theorem 1, if one desires to show absence of eigenvalues (or absolute continuity of the spectrum in the self-adjoint case), then the task should be to show existence of a quasimomentum $k \in \mathbb{C}^{d}$ such that the operator

$$
H(k, \mathbf{a}, V)=(D+k)^{2}+2 \mathbf{a}(x) \cdot(D+k)+V
$$

has zero kernel on the torus $\mathbb{T}^{d}$. We will prove the following facts.

Theorem 5. Let the magnetic potential a belong to $H^{s}\left(\mathbb{T}^{d}\right)$ for some $s>3 d / 2-1$ and satisfy the following conditions:

1. The average values of the components of a are equal to zero:

$$
\int_{\mathbb{T}^{d}} a_{j}(x) d x=0, \quad j=1, \ldots, d .
$$

2. For any $j, k=1, \ldots, d$ and $x, y \in \mathbb{R}^{d}$ the values of the matrix functions $a_{j}$ satisfy the commutation relation

$$
\left[a_{j}(x), a_{k}(y)\right]+\left[a_{k}(x), a_{j}(y)\right]=0 .
$$

Then there exist a constant $C>0$, a vector $e \in \mathbb{R}^{d}$, and a positive number $\beta \in \mathbb{R}$ such that

$$
\|H(k, \mathbf{a}, 0) u\|_{L_{2}} \geq C\left(|\rho|\|u\|_{L_{2}}+\|u\|_{H^{1}}\right)
$$

for any $k=(\beta+i \rho) e \in \mathbb{C}^{d}$ with sufficiently large $\rho \in \mathbb{R}$ and for any $u \in H^{2}\left(\mathbb{T}^{d}\right)$.

Remark 6. 1. Notice that self-adjointness of the matrices $a_{j}$ is not required.

2. The commutation relation (13) implies in particular the commutativity of the values of each of the functions $a_{j}(x)$, but not necessarily commutation between $a_{j}(x)$ and $a_{k}(y)$. Although commutativity of all the matrices $a_{j}(x)$ for different values of $j$ and $x$ is sufficient for (13), it is not necessary for the proof, and in fact the example of the Dirac operators leads to the case when such commutativity does not hold, while (13) still does. 
3. We presume that the condition (13) is probably not necessary for the theorem to hold. However, attempts to prove the statement without it stumble upon an analytic obstacle in the form of non-triviality of a vector bundle. So, one cannot rule out the possibility of surprises. We will discuss this further in Section 8 .

Theorem 5 leads to the immediate conclusion that the equation $H(k, \mathbf{a}, 0) u=0$ has no non-zero solutions on $\mathbb{T}^{d}$ for large values of $\rho$. Then, as in the previous section, Thomas' Theorem 1 implies that the operator $H(\mathbf{a}, 0)$ has no eigenvalues (and hence has absolutely continuous spectrum in the self-adjoint case). Including an electric potential $V$ that satisfies appropriate conditions is now a matter of technique (see [12] and [65]). For instance, using techniques described in [54, [37, and [65], this easily leads to the following result.

Theorem 7. Assume that the conditions of Theorem 5 are satisfied and that $V \in$ $L_{p, l o c}\left(\mathbb{R}^{d}\right)$ with $p=2$ for $d=2$ and $p>d-1$ for $d \geq 3$. Then the periodic operator $H(\mathbf{a}, V)$ has no eigenvalues in $L_{2}\left(\mathbb{R}^{d}\right)$.

Notice that self-adjointness of any of the potentials is not assumed. In the selfadjoint case this implies

Theorem 8. If the conditions of Theorem 7 are satisfied and the matrices $a_{j}(x)$ and $V(x)$ are self-adjoint for all $j$ and $x$, then the spectrum of the operator $H(\mathbf{a}, V)$ is absolutely continuous.

In fact, the conditions imposed on the magnetic and electric potentials are too restrictive. Using techniques of Section 5 in [12, one can extend the above results to the case when $V \in L_{p, l o c}$ with $p>1$ when $d=2, p=d / 2$ for $d=3$ and 4 , and $p=d-2$ when $d \geq 5$. In fact, for $d \geq 3$ the Lorentz spaces $L_{p, \infty}^{0}$ suffice [12]. We will not provide the proof of this extension here. One can refer to Section 5 in [12] for the method of its deduction from Theorem 5 . One can find the optimal conditions on the electric potential $V$ in terms of different function classes in [12] and [55]-58]. Although it is not clear yet how to optimize the conditions on the magnetic potential a for $d>2$, for $d=2$ the optimal conditions in $L_{p}$-classes are known - see the corresponding results and conjectures in [12], some new advances in [24, and discussion at the end of the paper.

\section{Sketch of the PROOF}

The approach we use is rather standard in microlocal analysis: microlocal reduction of the problem to a standard one, treating this model case, and construction of a parametrix using a partition of unity. This finally leads to the invertibility of the operator for large values of $\rho$. Due to periodicity, the dual space will be discrete.

Fourier series expansion transfers the consideration to functions defined on the dual lattice $2 \pi \mathbb{Z}^{d}$. The Fourier coefficients of a function $f$ on the torus $\mathbb{T}^{d}$ will be denoted by $\widehat{f}(m)$. We will need the function

$$
\Lambda(m, \rho)=\left(m^{2}+\rho^{2}\right)^{1 / 2}
$$

and the corresponding (pseudo-differential of first order) operator $\Lambda_{\rho}(D)$ on the torus $\mathbb{T}^{d}$ acting as

$$
\left(\Lambda_{\rho}(D) f\right) \widehat{)}(m)=\Lambda(m, \rho) \widehat{f}(m) .
$$


Notice that

$$
\left\|\Lambda_{\rho}(D) f\right\|_{L_{2}} \sim|\rho|\|u\|_{L_{2}}+\|u\|_{H^{1}} .
$$

Consider the case of zero electric potential first. It is known (see [12], 14], 65], and Section (6) how to include then the electric potential.

Let us introduce some notations:

$$
\begin{gathered}
H=-\Delta+2 \mathbf{a}(x) \cdot D, \\
H_{0}=-\Delta, \\
H(k)=(D+k)^{2}+2 \mathbf{a}(x) \cdot(D+k),
\end{gathered}
$$

and

$$
H_{0}(k)=(D+k)^{2} .
$$

We consider $H(k)$ and $H_{0}(k)$ as operators on the torus $\mathbb{T}^{d}$. Let $k=2 \pi(i \rho+\beta) e \in$ $\mathbb{C}^{d}$, where $\beta \in \mathbb{R}$ is fixed, $\rho \in \mathbb{R}$ is arbitrarily large, and $e \in \mathbb{R}^{d}$. The idea behind the proof of the estimate (14) is the following. We will construct a family of operators $R_{\rho}$ such that

$$
R_{\rho} H(k) \Lambda_{\rho}^{-1}=I+T_{\rho},
$$

where

$$
\left\|T_{\rho}\right\|_{L_{2} \rightarrow L_{2}} \rightarrow 0, \quad \rho \rightarrow \infty
$$

and

$$
\left\|R_{\rho}\right\|_{L_{2} \rightarrow L_{2}} \leq \text { const, } \quad \rho \rightarrow \infty .
$$

This for large $\rho$ would imply (14):

$$
\begin{gathered}
|\rho|\|u\|_{L_{2}}+\|u\|_{H^{1}} \sim\left\|\Lambda_{\rho}(D) f\right\|_{L_{2}}=\left\|\left(I+T_{\rho}\right)^{-1} R_{\rho} H(k) \Lambda_{\rho}^{-1} \Lambda_{\rho}(D) f\right\|_{L_{2}} \\
\leq C\|H(k) f\|_{L_{2}} .
\end{gathered}
$$

Construction of the approximate inversion operators $R_{\rho}$ is done locally and then pasted together using an appropriate partition of unity. Let us add some details. The symbol of the operator $H_{0}(k)$ is

$$
H_{0}(k, m)=(2 \pi m+k)^{2}=4 \pi^{2}\left[(m+\beta e)^{2}-\rho^{2}+2 i \rho e \cdot(m+\beta e)\right] .
$$

We consider this as the principal symbol of the full operator $H(k)$. Notice that we include some lower order differential terms with parameter $k$ into the principal symbol, which is rather standard when working with operators with parameters (see for instance [1]). The main difficulty is that the resulting problem with a parameter is not elliptic in the sense of [1]. The zero set of this symbol is

$$
Z_{\rho}=\left\{m \mid(m+\beta e)^{2}=\rho^{2}, e \cdot(m+\beta e)=0\right\} \subset \mathbb{R}^{d} .
$$

Now the situation looks different away from the set $Z_{\rho}$ and close to it. Namely, sufficiently far away from this set the principal part will dominate the magnetic one, and hence an approximate inverse can be constructed by neglecting the magnetic part. One ends up using the estimates of Section [2. However, close to $Z_{\rho}$ the magnetic potential part becomes of comparable strength with the principal one. Then one chooses a suitable finite covering of $Z_{\rho}$ by sets of diameter $\rho^{\delta}$ with an appropriately chosen $\delta \in(0,1)$. In each of the open sets of this covering the operator 
$H(k) \Lambda_{\rho}^{-1}$ is split into two parts by linearizing its symbol at a point. The linear term is a model first order differential operator. It happens to be the generalized Cauchy-Riemann operator

$$
\frac{\partial}{\partial \bar{z}}+F(z)
$$

on the complex plane, where the plane arises as a rational plane in $\mathbb{R}^{d}$, and the matrix function $F(z)$ is periodic and commutative. The remainder can be estimated away in the sense that approximate inversion of the model operator immediately leads to an approximate inverse for the whole sum. So, locally the problem is reduced to inverting the operator (15) on the torus. The nice trick that does exactly that was invented by A. Sobolev [65]. The idea is that under an appropriate choice of the vector $e$ used above for defining the quasimomentum $k$, one can gauge away most of the magnetic potential $a$ in (15). Hence the problem is reduced to the Cauchy-Riemann operator on the torus, which is an easily treatable case. As the result of this procedure, we have a set of local approximate inverse operators $R_{k, j}$ with suitable estimates. Then an appropriate partition of unity $\sum \phi_{j}=1$ in the dual space finishes the job. Namely, let

$$
\psi_{j}=\sum \phi_{k}
$$

where the sum runs over all values of $k$ such that the supports of $\phi_{j}$ and $\phi_{k}$ intersect, and define the operator

$$
R(k)=\sum_{j} \phi_{j}(k) R_{k, j} \psi_{j}(k)
$$

It is constructed in such a way that

$$
R(k) H(k) \Lambda_{\rho}^{-1}=I+T(k),
$$

where

$$
\lim _{\rho \rightarrow \infty}\|T(k)\|=0 .
$$

This proves (14) and all the theorems stated in the previous section.

We claim that all types of periodic operators for which absolute continuity of the spectrum has been proven so far (Schrödinger, Dirac, Maxwell, etc.) can be reduced to the case treated in Theorem 7 This will be shown in Section 7 However, the case of a magnetic potential a not satisfying (13) is also of importance and still remains a challenge. We expect that the absolute continuity must also hold without assuming (13) (see however the discussion in Section 8).

\section{Proof of the main estimate}

We will now present the proof of Theorem 5

Let us denote by $H_{0}$ the principal part $-\Delta$ of the operator $H=-\Delta+2 \mathbf{a}(x) \cdot D$ and by $H_{0}(m)$ its symbol $4 \pi^{2} m^{2}$. As before, the Fourier coefficients of a function $f$ on the torus will be denoted by $\widehat{f}(m)$. We use the function $\Lambda(m, \rho)=\left(m^{2}+\rho^{2}\right)^{1 / 2}$ introduced above and the corresponding (pseudo-differential of first order) operator $\Lambda_{\rho}(D)$ on the torus. 
Let $n \in \mathbb{Z}^{d}$ be a primitive integer vector, i.e. such that $\lambda n \notin \mathbb{Z}^{d}$ for $\lambda \in(0,1)$. The particular choice of $n$ will be specified later. We denote

$$
e=\frac{n}{|n|} \in \mathbb{R}^{d}
$$

and

$$
\beta=\frac{1}{2|n|} .
$$

Consider the quasimomentum $k=2 \pi(\beta+i \rho) e \in \mathbb{C}^{d}$, where $\rho>0$. This choice of the quasimomentum guarantees invertibility of the operator $H_{0}(D+k)$ on the torus. This follows from the direct calculation of the symbol:

$$
H_{0}(2 \pi m+k)=(2 \pi m+k)^{2}=4 \pi^{2}\left[(m+\beta e)^{2}-\rho^{2}+2 i \rho e \cdot(m+\beta e)\right] .
$$

Due to the specific choice of $e$, we now get

$$
\begin{aligned}
& \left|H_{0}(2 \pi m+k)\right| \geq\left|\operatorname{Im} H_{0}(2 \pi m+k)\right| \\
= & 8 \pi^{2} \rho|n|^{-1}|n \cdot m+0.5| \geq 4 \pi^{2} \rho|n|^{-1} .
\end{aligned}
$$

This proves invertibility. Besides, the norm in $L_{2}\left(\mathbb{T}^{2}\right)$ of the inverse operator is bounded from above by $|n|\left(4 \pi^{2} \rho\right)^{-1}$.

The characteristic set, or the set of degeneration of the symbol, is introduced as in the previous section:

$$
Z_{\rho}=\left\{\xi \mid(\xi+\beta e)^{2}=\rho^{2}, e \cdot(\xi+\beta e)=0\right\} .
$$

It can be represented as the intersection of the sphere

$$
S_{\rho}=\left\{\xi \mid(\xi+\beta e)^{2}=\rho^{2}\right\}
$$

with the hyperplane

$$
P=\{\xi \mid e \cdot(\xi+\beta e)=0\} .
$$

5.1. Inverse operator away from the characteristic set. Let us fix a number $\delta \in(0,1 / 2)$. Consider the "good" set

$$
U_{\rho, 0}=\left\{m \mid \operatorname{dist}\left(m, Z_{\rho}\right) \geq \rho^{\delta}\right\} .
$$

We call this set "good", since one can show that on this set the principal part dominates the magnetic terms. Namely, the following simple lemma holds.

Lemma 9. There exist operators $R_{\rho, 0}$ and $T_{\rho, 0}$ such that for any function $\psi \in$ $L_{2}\left(\mathbb{T}^{d}\right)$ with support of $\widehat{\psi}$ in $U_{\rho, 0}$ one has

$$
R_{\rho, 0}(k) H(k) \Lambda_{\rho}^{-1} \psi=\psi+T_{\rho, 0} \psi,
$$

where for $\rho \rightarrow \infty$ one has

$$
\left\|R_{\rho, 0}\right\|_{L_{2} \rightarrow L_{2}} \leq C, \quad\left\|T_{\rho, 0}\right\|_{L_{2} \rightarrow L_{2}} \rightarrow 0 .
$$

Proof of the lemma. Consider the following representation:

$$
H(k) \Lambda_{\rho}(D)^{-1}=H_{0}(k) \Lambda_{\rho}(D)^{-1}+A(k),
$$

where

$$
A(k)=2 \mathbf{a} \cdot(D+k) \Lambda_{\rho}(D)^{-1}
$$


Let us notice that the magnetic potential is bounded, so there is an estimate, uniform with respect to all parameters involved:

$$
\|A(k)\|_{L_{2} \rightarrow L_{2}} \leq \text { const. }
$$

Let us now look at the constant coefficients part $H_{0}(D+k) \Lambda_{\rho}(D)^{-1}$. Its symbol is

$$
4 \pi^{2}\left[(m+\beta e)^{2}-\rho^{2}+2 i \rho e \cdot(m+\beta e)\right]\left(m^{2}+\rho^{2}\right)^{-1 / 2} .
$$

We need to estimate this symbol from below on the set $U_{\rho, 0}$. Pulling $\rho$ out of the symbol, we reduce the problem to estimating from below the function

$$
\left[q^{2}-1+2 i e \cdot q\right]\left(q^{2}+1\right)^{-1 / 2}
$$

when $q=\rho^{-1}(m+\beta e)$ is at a distance at least $\rho^{\delta-1}$ from the set $Z_{1}$. We claim that in this case

$$
\left|\left[q^{2}-1+2 i e \cdot q\right]\left(q^{2}+1\right)^{-1 / 2}\right| \geq C \rho^{\delta-1} .
$$

Indeed, either $q$ is at a distance at least $\rho^{\delta-1}$ from the unit sphere centered at the origin, or it is at a distance at least $\rho^{\delta-1}$ from the hyperplane $e \cdot q=0$ being at the same time at a distance no more than $\rho^{\delta-1}$ from the unit sphere. In the first case the real part of our expression can be estimated from below by $\rho^{\delta-1}$. In order to show this, it is sufficient to rewrite the real part as

$$
(|q|-1)(|q|+1)\left(q^{2}+1\right)^{-1 / 2},
$$

which gives a lower bound of the kind we need. In the second case we will estimate the imaginary part. Namely, we know that $|q| \in\left(1-\rho^{\delta-1}, 1+\rho^{\delta-1}\right)$ and $|e \cdot q| \geq$ $\rho^{\delta-1}$. Then in the expression

$$
2|e \cdot q|\left(q^{2}+1\right)^{-1 / 2}
$$

the term $\left(q^{2}+1\right)^{-1 / 2}$ behaves as 1 , and the rest is estimated from below by $\rho^{\delta-1}$. The estimate (20) implies that one can choose an operator $R_{\rho, 0}$ as follows:

$$
R_{\rho, 0}=\left(H_{0}(k) \Lambda_{\rho}^{-1}\right)^{-1} \chi(D),
$$

where $\chi$ is a function which is equal to 1 on $U_{\rho, 0}$ and is supported in a slightly larger set of the same type. Then on functions $\psi$ supported in $U_{\rho, 0}$ one has

$$
R_{\rho, 0} H(k) \Lambda_{\rho}^{-1} \psi=\psi+T_{\rho, 0} \psi,
$$

where

$$
T_{\rho, 0}=\left(H_{0}(k) \Lambda_{\rho}^{-1}\right)^{-1} \chi A(k) .
$$

The estimates (18) now follow from (19) and (20). This proves the lemma.

5.2. Local behavior on the characteristic set. Now we have to deal with the "bad" set, i.e. the complement to $U_{\rho, 0}$. Let us choose a point $\xi$ on the characteristic set $Z_{\rho}$ :

$$
(\xi+\beta e)^{2}=\rho^{2}, \quad e \cdot(\xi+\beta e)=0 .
$$

The conditions (21) are equivalent to the representation of the vector $\xi$ in the form

$$
\xi=-\beta e+\rho l,
$$

where $l \in \mathbb{R}^{d}$ is a unit vector orthogonal to $e$. We will restrict consideration only to the rational points $l$, so the line $t l$ contains an integer point. This reduces the choice of $\xi$ to a dense subset in $Z_{\rho}$, which will be sufficient for our purpose. Let 
us also choose a neighborhood $V_{l, \rho}$ of the point $\xi$ such that its diameter is bounded by $C \rho^{\delta}$ with an absolute constant $C$ and a finite set of such neighborhoods covers the complement of $U_{\rho, 0}$ with a finite (independent of $\rho$ ) multiplicity. The exact choice of these sets will be specified later. Consider the operator $H(k) \Lambda_{\rho}(D)^{-1}$. Its symbol is

$$
h_{k}(m, x)=(m+k)^{2}\left(m^{2}+\rho^{2}\right)^{-1 / 2}+2 \mathbf{a}(x) \cdot(m+k)\left(m^{2}+\rho^{2}\right)^{-1 / 2} .
$$

This operator acts as $O p\left(h_{k}\right)$. This means that in order to apply this operator to a periodic function one expands the function into the Fourier series (with $m$ as the dual variable), then multiplies its terms by $h_{k}(m, x)$, and sums the series.

We are now going to linearize the symbol at the point $m=\xi$ in the neighborhood $V_{l, \rho}$. The linearization can be done by applying the Taylor expansion, or alternatively by a simple algebraic calculation that we are going to perform. In $V_{l, \rho}$ the function $\left(m^{2}+\rho^{2}\right)^{-1 / 2}$ behaves as $C \rho^{-1}$. Any point $m$ in this neighborhood can be represented as $m=-\beta e+\rho l+\nu$, where $|\nu| \leq C \rho^{\delta}$. Using this representation and the equality $k=(\beta+i \rho) e$, we get

$$
\begin{gathered}
(m+k)^{2}+2 \mathbf{a}(x) \cdot(m+k) \\
=(\rho(i e+l)+\nu)^{2}+2(\rho(i e+l)+\nu) \cdot \mathbf{a} \\
=2 \rho(i e+l) \cdot(\nu+\mathbf{a}(x))+\Phi=2 \rho(i e+l) \cdot(m+\beta e-\rho l+\mathbf{a}(x))+\Phi,
\end{gathered}
$$

where the symbol

$$
\Phi(m, x)=\nu^{2}+2 \nu \cdot \mathbf{a}
$$

satisfies in $V_{l, \rho}$ the estimate

$$
|\Phi(m, x)| \leq C \rho^{2 \delta} .
$$

Hence, in the same neighborhood

$$
\left|\Phi(m, x)\left(m^{2}+\rho^{2}\right)^{-1 / 2}\right| \leq C \rho^{2 \delta-1} .
$$

Now the symbol $h_{k}(m, x)$ can be represented as

$$
h_{k}(m, x)=\sqrt{2}(i e+l) \cdot(m+\beta e-\rho l+\mathbf{a}(x))+\Psi(m, x),
$$

where the symbol

$$
\begin{aligned}
\Psi= & 2 \rho(i e+l) \cdot(m+\beta e-\rho l+\mathbf{a}(x))\left(\left(m^{2}+\rho^{2}\right)^{-1 / 2}-1 /(\sqrt{2} \rho)\right) \\
& +\Phi(m, x)\left(m^{2}+\rho^{2}\right)^{-1 / 2}
\end{aligned}
$$

satisfies for large values of $\rho$ the estimate

$$
|\Psi(m, x)| \leq C \rho^{2 \delta-1} .
$$

The constants in the last two estimates are absolute.

As the result, we can conclude that the operator $H(k) \Lambda_{\delta}(D)^{-1}$ can be represented on functions $\psi$ whose Fourier coefficients are supported in $V_{l, \rho}$ as

$$
H(k) \Lambda_{\delta}(D)^{-1}=\sqrt{2} H_{\xi}+\Psi,
$$

where $H_{\xi}=O p\left(h_{\xi}\right)$ has the symbol

$$
h_{\xi}(m)=(i e+l) \cdot(m+\beta e-\rho l+\mathbf{a}(x))
$$


and $\Psi=O p(\Psi(m, x))$ obeys the estimate

$$
|\Psi(m, x)| \leq C \rho^{2 \delta-1}
$$

on $V_{l, \rho}$. We remind the reader that here $\xi=-\beta e+\rho l$.

Let us notice that the symbol (24) corresponds to the first order differential operator on the torus of the form $\partial / \partial \bar{z}+F(z)$, if we identify the $l$-e plane with the complex plane $\mathbb{C}$. Here $F(z)=(i e+l) \cdot \mathbf{a}(x)$ is a periodic matrix function with zero average. This is the model operator that we will have to deal with.

Lemma 10. The values of the matrix function $F=(i e+l) \cdot \mathbf{a}(x)$ commute.

Proof of the lemma. Let us denote $(i e+l)=\left(s_{1}, \ldots, s_{d}\right)$. Then

$$
(i e+l) \cdot \mathbf{a}(x)=\sum_{j} s_{j} a_{j}(x) .
$$

Now direct calculation shows that the condition (13) implies commutativity of these matrices for different values of $x$. Indeed,

$$
\begin{aligned}
& {[F(x), F(y)]=\left[\sum_{j} s_{j} a_{j}(x), \sum_{k} s_{k} a_{k}(y)\right]=\sum_{1 \leq j, k \leq d} s_{j} s_{k}\left[a_{j}(x), a_{k}(y)\right] } \\
= & \sum_{1 \leq j \leq d} s_{j}^{2}\left[a_{j}(x), a_{j}(y)\right]+\sum_{1 \leq j<k \leq d} s_{j} s_{k}\left(\left[a_{j}(x), a_{k}(y)\right]+\left[a_{k}(x), a_{j}(y)\right]\right) .
\end{aligned}
$$

Now all the terms in the last two sums are equal to zero due to (13).

The following lemma enables us to neglect the remainder term $\Psi$ in (23) if $\delta$ is small enough.

Lemma 11. There exists a constant $C$ such that for any function $v$ which has its Fourier series supported in $V_{l, \rho}$ the following estimate holds:

$$
\|\Psi v\|_{L_{2}} \leq C \rho^{(d+4) \delta / 2-1}\|v\|_{L_{2}} .
$$

In particular, if

$$
\delta<2 /(d+4),
$$

then the operator norm of $\Psi$ on the subspace of such functions tends to zero when $\rho \rightarrow \infty$.

The proof of the lemma is straightforward. Indeed,

$$
|(\Psi v)(x)|^{2}=\left|\sum_{m \in V_{l, \rho}} \Psi(m, x) \widehat{v}(m) e^{2 \pi i m \cdot x}\right|^{2} .
$$

Now the Cauchy-Schwarz inequality together with (25) and an estimate of the size of $V_{l . \rho}$ by $\rho^{d \delta}$ finish the job.

This lemma and expansion (23) show that it is sufficient for our purpose to invert the model operator $H_{\xi}$ with uniform estimates on the norm of $\left(H_{\xi}\right)^{-1}$ on functions Fourier-supported in $V_{l, \rho}$. 
5.3. Inversion of the model operator. In this section our goal is to construct the inverse of the model inhomogeneous $\bar{\partial}$-operator described above, and to get estimates for it. This will be used to construct a local approximate inverse operator in $V_{l, \rho}$ and then a global approximate inverse. In order to make the consideration better understandable, we will start with the simplest case and move toward the most general one. In each case we will have to make an appropriate choice of the vector $e$, which so far has been arbitrary.

5.3.1. Zero magnetic potential. In this case $\mathbf{a}=0$ (which is the situation considered by Thomas [68]). Then the model operator (which we will denote by $H_{\xi}^{0}$ ) has constant coefficients. The imaginary part of its symbol

$$
(i e+l) \cdot(m+\beta e-\rho l)
$$

is equal to

$$
e \cdot(m+\beta e)
$$

(recall that the vectors $e$ and $l$ are orthogonal). Let us now choose any non-zero vector $n \in \mathbb{Z}^{d}$ and denote $e=n /|n|$. We assume, as before, that $\beta=1 /(2|n|)$. Then we have an estimate of the symbol from below:

$$
\begin{gathered}
|(i e+l) \cdot(m+\beta e-\rho l)| \geq|e \cdot(m+\beta e)| \\
=\frac{1}{|n|}\left|m \cdot n+\frac{1}{2}\right| \geq \frac{1}{2|n|},
\end{gathered}
$$

and hence

$$
\left\|H_{\xi}^{0} \psi\right\|_{L_{2}} \geq \frac{1}{2|n|}\|\psi\|_{L_{2}} .
$$

This means that we can use as an approximate inverse the operator

$$
R_{l, \rho}=\frac{1}{\sqrt{2}}\left(H_{\xi}^{0}\right)^{-1}
$$

on functions with Fourier support inside $V_{l, \rho}$. Indeed, we see that $\left\|R_{l, \rho}\right\| \leq$ const and

$$
\frac{1}{\sqrt{2}}\left(H_{\xi}^{0}\right)^{-1} H(D+k) \Lambda_{\delta}(D)^{-1}=\left(H_{\xi}^{0}\right)^{-1}\left(H_{\xi}^{0}+\Psi\right)=I+\left(H_{\xi}^{0}\right)^{-1} \Psi
$$

Here the operator $T=R \Psi=\frac{1}{\sqrt{2}}\left(H_{\xi}^{0}\right)^{-1} \Psi$ obviously has the norm tending to zero for large values of $\rho$.

5.3.2. Small magnetic potential. Let us denote $\|\mathbf{a}\|=\max _{x \in \mathbb{T}^{d}, j=1, \ldots, d}\left\|a_{j}(x)\right\|$, where $\left\|a_{j}(x)\right\|$ is the operator norm of the matrix $a_{j}(x)$ in the Euclidean space $\mathbb{C}^{n}$. We will assume that $\|\mathbf{a}\|$ is sufficiently small (it will be clear from what follows how small it should be). This is the situation considered in 31]. Let us choose $n=e=(1,0, \ldots, 0)$ and $\beta=1 / 2$. We can consider our model operator $H_{\xi}+\Psi$ as a perturbation of the free one:

$$
H_{\xi}+\Psi=H_{\xi}^{0}+O p(2 \pi(i e+l) \cdot \mathbf{a})+\Psi .
$$

If $\|\mathbf{a}\|$ is small enough and $\rho$ is large enough, we conclude that

$$
\|T\|=\left\|\left(H_{\xi}^{0}\right)^{-1}(O p(2 \pi(i e+l) \cdot \mathbf{a})+\Psi)\right\|<1,
$$


and hence we can define the inverse operator

$$
R_{l, \rho}=(I+T)^{-1}\left(H_{\xi}^{0}\right)^{-1} .
$$

It is clear that uniform norm estimates from above hold for $R_{l, \rho}$.

5.3.3. $2 D$ case. The two-dimensional case was settled in 8]. We will show here how it fits into the general scheme. Let us assume that $d=2$. In this case the only vector $m \in \mathbb{R}^{d}$ such that $(i e+l) \cdot m=0$ is $m=0$. Since the magnetic potential has zero average and the values of $(i e+l) \cdot \mathbf{a}$ commute, one can gauge away the magnetic potential completely. Namely, let $\phi(x)$ be a commutative matrix function of $x \in$ $\mathbb{R}^{2}$, periodic with respect to the lattice $\mathbb{Z}^{2}$, that also commutes with all values of $(i e+l) \cdot \mathbf{a}$. We can commute the model operator $H_{\xi}=(i e+l) \cdot(D+\beta e-\rho l)+(i e+l) \cdot \mathbf{a}$ with the exponent $e^{i \phi(x)}$ :

$$
e^{-i \phi(x)} \circ H_{\xi} \circ e^{i \phi(x)}
$$

This, due to the commutativity of the values of all matrix functions involved, produces the operator

$$
(i e+l) \cdot(D+\beta e-\rho l)+(i e+l) \cdot(\mathbf{a}+\nabla \phi) .
$$

Assume now that we choose a function $\phi$ such that

$$
(i e+l) \cdot \nabla \phi=-(i e+l) \cdot \mathbf{a} .
$$

(One can easily identify this equality with an inhomogeneous $\bar{\partial}$-equation with respect to $\phi$ in the $e-l$ plane considered as the complex plane $\mathbb{C}$.) This is possible if a has zero average (which we do assume). Namely, the Fourier coefficients of the function $\phi$ can be found as

$$
\widehat{\phi}(m)=-\frac{(i e+l) \cdot \widehat{\mathbf{a}}(m)}{(i e+l) \cdot m}
$$

(we use the fact that $\widehat{\mathbf{a}}(0)=0$ ). It is clear from the construction of $\phi(x)$ and the commutativity of the matrices $(i e+l) \cdot \widehat{\mathbf{a}}(m)$ that the matrix function $\phi$ is commutative and commutes with all matrices $(i e+l) \cdot \mathbf{a}(x)$. This enables us to apply the gauge transform as above, after which the magnetic potential goes away and we are again in the situation of the zero magnetic potential. In other words,

$$
\left(H_{\xi}\right)^{-1}=e^{i \phi(x)}\left(H_{\xi}^{0}\right)^{-1} e^{-i \phi(x)} .
$$

The formula (29) also provides an uniform (with respect to $\rho$ and $l$ ) estimate

$$
\|\phi\|_{L_{\infty}\left(\mathbb{T}^{2}\right)} \leq C_{\varepsilon}\|\mathbf{a}\|_{H^{\varepsilon}\left(\mathbb{T}^{2}\right)}
$$

for any positive $\varepsilon$. This gives uniform estimates for the $L_{2}$-norm of the gauge transform. Using now the considerations of the case when $\mathbf{a}=\mathbf{0}$, we end up with a uniform estimate from above for $R_{l, \rho}=\left(H_{\xi}\right)^{-1}$.

5.3.4. General case. The situation in dimensions higher than two (first considered in the scalar self-adjoint case in [65]) is definitely much trickier. One can see where the difficulty lies in comparison with $2 D$. Namely, when $d>2$, the division problem (29) cannot be resolved for an arbitrary potential a with zero average. The reason is that the denominator in (29) has many zeros besides $m=0$. This means that the magnetic potential cannot be gauged away completely. The clever trick invented by A. V. Sobolev is to use a gauge transform for "almost" eliminating the magnetic 
potential, rather than for its complete removal as was done for $d=2$. Let us describe this method in detail.

One needs to find an integer vector $n$ (which will determine the vector $e=n /|n|$ and the number $\beta=1 / 2|n|$ as before) and a large positive number $L$ such that if in the Fourier expansion of the magnetic potential a we keep only the finite sum up to $L$, then the resulting approximation to the potential

$$
\mathbf{a}_{L}(x)=\sum_{|m| \leq L} \widehat{\mathbf{a}}(m) e^{2 \pi i m \cdot x}
$$

can be gauged away according to (29). If additionally the norm of the remainder $\mathbf{a}-\mathbf{a}_{L}$ were sufficiently small, then we would have reduced the problem to the case of a small magnetic potential. So, now we need to find a vector $e=n /|n|$ and a number $L>0$ such that

i) $(i e+l) \cdot m \neq 0$ for any rational vector $l$ orthogonal to $e$ and $m \neq 0,|m|<L$;

ii) || $\mathbf{a}-\mathbf{a}_{L} \|<\theta /|n|$, where $\theta$ is a given small constant. We would also like to have uniform control over the norm $\left\|\mathbf{a}_{L}\right\|_{H \varepsilon}$.

If this is done, then according to (29) we can find a gauge transform with the phase $\phi(x)$ such that it eliminates the $\mathbf{a}_{L}$ part of the magnetic potential. Besides, $\|\phi\|_{\infty} \leq C(L, \mathbf{a})$, where $C(L, \mathbf{a})$ depends on $L$ and $\mathbf{a}$ only (and in particular does not depend on $\rho$ and $l$ ). This reduces the problem to the case of a small potential $\mathbf{a}-\mathbf{a}_{L}$, which we have already treated. Let us notice that "small" means less than $C \beta=\theta /|n|$ with some absolute constants $C$ and $\theta$. In other words, choosing a longer integer vector $n$ imposes much stronger requirement on the part of the potential that must be gauged away.

Let us implement this program. The idea is that for an appropriately chosen integer direction $n$ all integer vectors $m$ that are orthogonal to $n$ have a large norm. This means that the plane $e \cdot m=0$ (and hence $(i e+l) \cdot m=0$ ) hits the points of the dual lattice very far from the origin (i.e., for $|m|>L$ with a large $L$ ). If this is true, then the "tail" $\mathbf{a}-\mathbf{a}_{L}$ will be small. This requires, however, some estimates, which we are going to present. The first step is to discuss the relation between $|n|$ and $L$.

Lemma 12 ([65]). For any integer $L>0$ the equation

$$
m_{1}+m_{2} L+\ldots+m_{d} L^{d-1}=0
$$

has no non-zero integer solutions $m=\left(m_{1}, \ldots, m_{d}\right)$ with $\left|m_{j}\right|<L / 2$. Moreover, if $m \neq 0$ and $\left|m_{j}\right|<L / 2$, then the following estimate holds:

$$
\left|m_{1}+m_{2} L+\ldots+m_{d} L^{d-1}\right| \geq 1 .
$$

Proof of the lemma. Let $m \neq 0$ and $\left|m_{j}\right|<L / 2$. Let $m_{k}(k \geq 1)$ be the first non-zero component among $m_{1}, \ldots, m_{d}$. Then

$$
\begin{aligned}
& \left|m_{1}+m_{2} L+\ldots+m_{d} L^{d-1}\right|=\left|m_{k} L^{k-1}+\ldots+m_{d} L^{d-1}\right| \\
= & \left|L^{k}\left(\frac{m_{k}}{L}+\left(m_{k+1}+\ldots+m_{d} L^{d-k}\right)\right)\right| \geq L^{k} \frac{1}{L}=L^{k-1} \geq 1 .
\end{aligned}
$$

This proves both statements of the lemma.

We now choose the integer vector $n=\left(1, L, L^{2}, \ldots, L^{d-1}\right)$, where $L$ is a large integer (note that $|n| \sim L^{d-1}$ ). Then the lemma above shows that all non-zero 
integer solutions of the equation $m \cdot n=0$ lie outside the ball of radius $L / 2 \sim$ $C|n|^{1 /(d-1)}$.

Consider the Fourier expansion of the magnetic potential:

$$
\mathbf{a}(x)=\sum \widehat{\mathbf{a}}(m) e^{2 \pi i m \cdot x}
$$

and assume that $\sum|| \widehat{\mathbf{a}}(m)|||m|^{\mu}<\infty$ for some $\mu$. Then

$$
\sum_{|m|>L}\|\widehat{\mathbf{a}}(m)\| \leq L^{-\mu} \sum_{|m|>L}|m|^{\mu}\|\widehat{\mathbf{a}}(m)\|=o\left(L^{-\mu}\right), \quad L \rightarrow \infty .
$$

If now $|n|=O\left(L^{\mu}\right)$, we see that

$$
|n| \sum_{|m|>L}\|\widehat{\mathbf{a}}(m)\| \rightarrow 0, \quad L \rightarrow \infty .
$$

Now the last lemma shows that if $\sum\|\widehat{\mathbf{a}}(m)\||m|^{d-1}<\infty$ (in particular if $\mathbf{a} \in$ $H^{s}\left(\mathbb{T}^{d}\right)$ for some $\left.s>3 d / 2-1\right)$, our requirement ii) on smallness of the residual $\mathbf{a}_{r}=\mathbf{a}-\mathbf{a}_{L}$ is satisfied. Here $\mathbf{a}_{L}$ is defined as in (30).

Our goal now is to gauge away the part $(i e+l) \cdot \mathbf{a}_{L}$ of the potential in the model operator. This is possible if the vector $e=n /|n|$ is chosen as above. Namely, let us again look for a periodic commutative (and commuting with the matrices $(i e+l) \cdot \mathbf{a}$ ) matrix function $\phi(x)$ such that

$$
(i e+l) \cdot \nabla \phi=-(i e+l) \cdot \mathbf{a}_{L} .
$$

This is possible according to Lemma 10 and formula (29), since we assume that $\widehat{\mathbf{a}}_{L}(0)=\widehat{\mathbf{a}}(0)=0$ and that $\widehat{\mathbf{a}}_{L}(m)=0$ whenever $(i e+l) \cdot m=0$. Besides, the estimate (31) together with the definition of the vector $e$ implies that

$$
\|\phi\|_{L_{\infty}} \leq C_{\varepsilon}\|\mathbf{a}\|_{H^{d / 2+\varepsilon}}
$$

for any positive $\varepsilon$, where the constant $C_{\varepsilon}$ does not depend on $l$ and $\rho$ (although it does depend on $n$ ).

Now we get the representation

$$
e^{-i \phi} H_{\xi} e^{i \phi}=H_{\xi}^{0}+(i e+l) \cdot \mathbf{a}_{r} .
$$

Choosing $L$ sufficiently large, we achieve smallness of $\mathbf{a}_{r}$ and hence, as explained in the case of a small potential, invertibility of $H_{\xi}^{0}+(i e+l) \cdot \mathbf{a}_{r}$ with a uniform estimate of the inverse. Then the operator

$$
R_{l, \rho}=e^{i \phi}\left(H_{\xi}^{0}+(i e+l) \cdot \mathbf{a}_{r}\right)^{-1} e^{-i \phi}
$$

inverts the operator $H_{\xi}$ and satisfies a uniform (with respect to $\rho$ and $l$ ) estimate from above.

Observe that the maximal smoothness of a required so far in the case $d>2$ is $\mathbf{a} \in H^{s}\left(\mathbb{T}^{d}\right)$ for some $s>3 d / 2-1$.

5.4. Global inverse operator and completion of the proof. The last step of the proof requires us to paste the local approximate inverse operators together, which is a rather standard procedure. Namely, let $\psi_{0} \in C_{0}^{\infty}\left(\mathbb{R}^{d}\right)$ be such that $\psi_{0} \geq 0, \psi_{0}(\xi)=1$ for $\xi \in[-0.5,0.5]^{d}$, and $\psi_{0}(\xi)=0$ for $\xi$ outside $[-1,1]^{d}$. Now set $\psi_{j}(\xi)=\psi_{0}(\xi-j)$ for $j \in \mathbb{Z}^{d}$, and $\chi_{j}=\psi_{j}\left(\sum \psi_{i}\right)^{-1}$. Then $\left\{\chi_{j}\right\}$ forms a partition of unity, the diameter of the support of $\chi_{j}$ does not exceed $2 \sqrt{d}$, and the multiplicity of the covering of the whole space by the supports of these functions is finite. 
Let us now fix $\delta \in(0,2 /(d+4))$ and define the stretched functions

$$
\chi_{\rho j}^{1}(\xi)=\chi_{j}\left(\rho^{-\delta} \xi\right)
$$

This new set of functions still forms a partition of unity, and, besides,

$$
\left|D^{\alpha} \chi_{\rho j}^{1}\right| \leq C_{\alpha} \rho^{-\delta|\alpha|}
$$

The support of $\chi_{\rho j}^{1}$ belongs to the cube

$$
Q_{\rho j}=\left\{\xi \mid \rho^{-\delta} \xi-j \in[-1,1]^{d}\right\} .
$$

Now we need a set of cut-off functions with a larger support. Namely, we define

$$
\chi_{\rho j}^{2}=\sum_{s} \chi_{\rho s}^{1},
$$

where the sum is taken over all values of $s$ for which the supports of $\chi_{\rho s}^{1}$ and $\chi_{\rho j}^{1}$ intersect. We obviously have estimates for $\chi_{\rho j}^{2}$ similar to those for $\chi_{\rho j}^{1}$. Besides,

$$
\chi_{\rho j}^{1} \chi_{\rho j}^{2}=\chi_{\rho j}^{1} .
$$

Consider the set of all values of $j$ such that the support of $\chi_{\rho j}^{2}$ is disjoint with the characteristic set $Z_{\rho}$. Then obviously the supports of the corresponding $\chi_{\rho j}^{1}$ belong to the set $U_{\rho, 0}$ as defined in Section 5.1. Hence, according to that section, in each of the corresponding cubes $Q_{\rho j}$ there exists an operator $R_{\rho j}$ such that $\left\|R_{\rho j}\right\|<C$ uniformly with respect to $\rho$,

$$
R_{\rho j} H(k) \Lambda_{\rho}^{-1} \psi=\psi+T_{\rho j} \psi
$$

on functions $\psi$ whose Fourier series is supported in $Q_{\rho j}$, and

$$
\left\|T_{\rho j}\right\| \rightarrow 0, \quad \rho \rightarrow \infty
$$

uniformly in $\rho$.

Consider now the values of $j$ for which the support of $\chi_{\rho j}^{2}$ does touch the characteristic set. Then, according to the considerations of the previous section, on each of the corresponding cubes $Q_{\rho j}$ there also exist operators $R_{\rho j}$ and $T_{\rho j}$ with the same properties.

We are now ready to construct an approximate inverse for the whole operator $H(k) \Lambda_{\rho}^{-1}$. We define it as follows:

$$
R_{\rho}=\sum_{j} \chi_{\rho j}^{1} R_{\rho j} \chi_{\rho j}^{2}
$$

where we denote the multiplier of the Fourier series by the function $\chi_{\rho j}^{1}$ by the same expression $\chi_{\rho j}^{1}$. Let us show that for large values of $\rho$ this is indeed an approximate inverse for $H(k)$.

Lemma 13. For sufficiently large values of $\rho$ the following relation holds:

$$
R_{\rho} H(k) \Lambda_{\rho}^{-1}=I+T_{\rho},
$$

where $\left\|T_{\rho}\right\|_{L_{2} \rightarrow L_{2}}<1$.

As explained before, this would prove Theorem 5 and the consequent results. 
Proof of the lemma. Let us compute the product $R_{\rho} H(k) \Lambda_{\delta}^{-1}$ :

$$
\begin{gathered}
R_{\rho} H(k) \Lambda_{\rho}^{-1}=\sum_{j} \chi_{\rho j}^{1} R_{\rho j} \chi_{\rho j}^{2} H(k) \Lambda_{\rho}^{-1} \\
=\sum_{j} \chi_{\rho j}^{1} R_{\rho j} H(k) \Lambda_{\rho}^{-1} \chi_{\rho j}^{2}+\sum_{j} \chi_{\rho j}^{1} R_{\rho j}\left[\chi_{\rho j}^{2}, H(k) \Lambda_{\rho}^{-1}\right] .
\end{gathered}
$$

Here $[A, B]$ denotes the commutator of the operators $A$ and $B$. The first sum in this expression is

$$
\left(\sum_{j} \chi_{\rho j}^{1}\right) I+\sum_{j} \chi_{\rho j}^{1} T_{\rho j} \chi_{\rho j}^{2}=I+T_{\rho} .
$$

Due to the finiteness of the multiplicity of the covering by the supports of $\chi_{\rho j}^{2}$, we conclude that

$$
\left\|T_{\rho}\right\|_{L_{2} \rightarrow L_{2}} \leq C \max _{j}\left\|T_{\rho j}\right\|_{L_{2} \rightarrow L_{2}} \rightarrow 0
$$

when $\rho \rightarrow \infty$. So, the only remaining task is to show that

$$
\left\|\sum_{j} \chi_{\rho j}^{1} R_{\rho j}\left[\chi_{\rho j}^{2}, H(k) \Lambda_{\rho}^{-1}\right]\right\|_{L_{2} \rightarrow L_{2}} \rightarrow 0
$$

when $\rho \rightarrow \infty$. Let us compute the commutator explicitly, taking into account that

$$
H(k)=(D+k)^{2}+2 \mathbf{a} \cdot(D+k) .
$$

The second order term $(D+k)^{2}$ has constant coefficients, and so commutes with $\chi_{\rho j}^{2}$, which is itself a constant coefficient operator. Hence the commutator can be expressed as follows:

$$
2\left[\chi_{\rho j}^{2}, \mathbf{a}\right] \cdot(D+k) \Lambda_{\rho}^{-1} .
$$

The operator $(D+k) \Lambda_{\rho}^{-1}$ is obviously bounded in $L_{2}$ uniformly with respect to $\rho$. Let $S_{\rho, j}=\left[\chi_{\rho j}^{2}, \mathbf{a}\right]$. Consider the block matrix of this operator in the basis of harmonics $e^{2 \pi i m \cdot x}$. The matrix of the operator of multiplication by a has entries $\left\{\widehat{\mathbf{a}}_{m-m^{\prime}}\right\}$, where as before $\widehat{\mathbf{a}}_{m}$ are the Fourier coefficients of $\mathbf{a}$. Then the matrix of $\left[\chi_{\rho j}^{2}, \mathbf{a}\right]$ has the entries

$$
\left(\chi_{\rho j}^{2}(m)-\chi_{\rho j}^{2}\left(m^{\prime}\right)\right) \widehat{\mathbf{a}}_{m-m^{\prime}}=\nabla \chi_{\rho j}^{2}\left(m^{\prime}+\theta\left(m-m^{\prime}\right)\right) \cdot\left(m-m^{\prime}\right) \widehat{\mathbf{a}}_{m-m^{\prime}} .
$$

Using the estimate (32), we conclude that

$$
\left|\left(\chi_{\rho j}^{2}(m)-\chi_{\rho j}^{2}\left(m^{\prime}\right)\right)\right| \leq C \rho^{-\delta}\left|m-m^{\prime}\right|
$$

with a constant $C$ independent of $j$ and $\rho$. Let us now assume that

$$
\sum_{m}|m|\left\|\widehat{\mathbf{a}}_{m}\right\|<\infty
$$

The estimate (34) follows for instance from the assumption that $\mathbf{a} \in H^{d / 2+1+\varepsilon}$. Let us also notice that for a fixed pair $\left(m, m^{\prime}\right)$ the number of indices $j$ for which $\left(\chi_{\rho j}^{2}(m)-\chi_{\rho j}^{2}\left(m^{\prime}\right)\right) \neq 0$ is bounded by a constant (for instance, by twice the multiplicity of the covering by the supports of $\chi_{\rho j}^{2}$ ). Consider now the infinite matrix $B$ with entries labeled by two indices $(j, m)$ and $m^{\prime}$ and given by the formula

$$
b_{(j, m), m^{\prime}}=\left(\chi_{\rho j}^{2}(m)-\chi_{\rho j}^{2}\left(m^{\prime}\right)\right)\left\|\widehat{\mathbf{a}}_{m-m^{\prime}}\right\| .
$$


We will estimate its norm as an operator from $l_{2}\left(\mathbb{Z}^{d}\right)$ into $l_{2}\left(\mathbb{Z}^{d} \times \mathbb{Z}^{d}\right)$ :

$$
B v_{(j, m)}=\sum_{m^{\prime}} b_{(j, m), m^{\prime}} v_{m^{\prime}} .
$$

In order to do so, we will estimate its operator norms in $l_{\infty}$ and $l_{1}$ first and then use interpolation. Estimates (33) and (34) imply that

$$
\begin{gathered}
\sum_{m^{\prime}}\left|b_{(j, m), m^{\prime}}\right|=\sum_{m^{\prime}}\left|\left(\chi_{\rho j}^{2}(m)-\chi_{\rho j}^{2}\left(m^{\prime}\right)\right)\right||| \widehat{\mathbf{a}}_{m-m^{\prime}} \| \\
\leq C \rho^{-\delta} \sum_{m^{\prime}}\left|m-m^{\prime}\right|\left|\widehat{\mathbf{a}}_{m-m^{\prime}}\right| \mid \leq C \rho^{-\delta} .
\end{gathered}
$$

This means that $\|B\|_{l_{\infty} \rightarrow l_{\infty}} \leq C \rho^{-\delta}$. Analogously, using the same estimates and the comment above about the number of indices $j$ for which $\left(\chi_{\rho j}^{2}(m)-\chi_{\rho j}^{2}\left(m^{\prime}\right)\right) \neq$ 0 , we get

$$
\begin{gathered}
\sum_{(j, m)}\left|b_{(j, m), m^{\prime}}\right|=\sum_{(j, m)}\left|\left(\chi_{\rho j}^{2}(m)-\chi_{\rho j}^{2}\left(m^{\prime}\right)\right)\right|\left\|\widehat{\mathbf{a}}_{m-m^{\prime}}\right\| \\
\leq C \rho^{-\delta} \sum_{m}\left|m-m^{\prime}\right| \mid \widehat{\mathbf{a}}_{m-m^{\prime}} \| \leq C \rho^{-\delta}
\end{gathered}
$$

We conclude that $\|B\|_{l_{1} \rightarrow l_{1}} \leq C \rho^{-\delta}$, and hence by interpolation

$$
\|B\|_{l_{2} \rightarrow l_{2}} \leq C \rho^{-\delta} \text {. }
$$

We are now able to estimate the norm of the operator $\sum_{j} \chi_{\rho j}^{1} R_{\rho j} S_{\rho j}$ in $L_{2}$. Namely, using the (bounded from above) multiplicity of the covering by the supports of $\chi_{\rho j}^{1}$ and the uniform boundedness of the operators $R_{\rho j}$, we conclude that

$$
\begin{aligned}
\left\|\sum_{j} \chi_{\rho j}^{1} R_{\rho j} S_{\rho j} u\right\|_{L_{2}}^{2} & \leq C \sum_{j}\left\|S_{\rho j} u\right\|^{2} \\
& =C \sum_{j, m}\left\|\sum_{m^{\prime}}\left(\chi_{\rho j}^{2}(m)-\chi_{\rho j}^{2}\left(m^{\prime}\right)\right) \widehat{\mathbf{a}}_{m-m^{\prime}} u_{m^{\prime}}\right\|^{2} \\
& \leq C \sum_{j, m}\left(\sum_{m^{\prime}}\left|\chi_{\rho j}^{2}(m)-\chi_{\rho j}^{2}\left(m^{\prime}\right)\right|\left\|\widehat{\mathbf{a}}_{m-m^{\prime}}\right\|\left\|u_{m^{\prime}}\right\|\right)^{2} \\
& =C\left\|B\left(\left\{\left\|u_{m}\right\|\right\}_{m \in Z^{d}}\right)\right\|_{l_{2}}^{2} .
\end{aligned}
$$

Now (35) implies

$$
\left\|\sum_{j} \chi_{\rho j}^{1} R_{\rho j} S_{\rho j} u\right\|_{L_{2}}^{2} \leq C \rho^{-2 \delta}\|u\|_{L_{2}}^{2} .
$$

This means that the norm of the operator $\sum_{j} \chi_{\rho j}^{1} R_{\rho j} S_{\rho j}$ tends to zero when $\rho \rightarrow \infty$. This concludes the proof of the lemma. 


\section{INCLUSION OF AN ELECTRIC POTENTIAL}

In this section we will explain how to derive Theorem[7 from Theorem 5 . Assume that $V$ belongs to the space $L_{p, l o c}\left(\mathbb{R}^{d}\right)$ with $p=2$ for $d=2$ and $p>d-1$ for $d \geq 3$. The way one can include the electric potential under these conditions is rather standard (see the proofs of Theorem XIII.10 in [54], Theorem 4.1.8 in [37], and Sections 3.5 and 7.2 in 65]). Namely, the Young and Hölder inequalities easily lead to the following estimate between the electric potential and the free terms:

$$
\|V u\|_{L^{t}\left(\mathbb{T}^{d}\right)} \leq C \rho^{-1+(d-1) / p}\|H(k, 0,0) u\|_{L^{t}\left(\mathbf{T}^{d}\right)}
$$

for any $u \in H^{2}\left(\mathbb{T}^{d}\right.$ ) (see the simple details in the proofs of Theorem 4.1.8 in 37] and Lemma 7.2 in [65]). Besides, one has the obvious estimate

$$
\|(H(k, 0,0)-H(k, \mathbf{a}, 0)) u\|_{L^{t}\left(\mathbf{T}^{d}\right)} \leq C\left\|\Lambda_{\rho}(D) u\right\|_{L^{t}\left(\mathbf{T}^{d}\right)} .
$$

This inequality, (36), (14), and the conditions on $p$ imply that

$$
\begin{gathered}
\|V u\|_{L^{t}\left(\mathbb{T}^{d}\right)} \leq \varepsilon\|H(k, 0,0) u\|_{L^{t}\left(\mathbf{T}^{d}\right)} \\
\leq \varepsilon\left(\|H(k, \mathbf{a}, 0) u\|_{L^{t}\left(\mathbf{T}^{d}\right)}+\|(H(k, 0,0)-H(k, \mathbf{a}, 0)) u\|_{L^{t}\left(\mathbf{T}^{d}\right)}\right) \\
\leq \varepsilon\left(\|H(k, \mathbf{a}, 0) u\|_{L^{t}\left(\mathbf{T}^{d}\right)}+C\left\|\Lambda_{\rho}(D) u\right\|_{L^{t}\left(\mathbf{T}^{d}\right)}\right) \\
\leq C \varepsilon\|H(k, \mathbf{a}, 0) u\|_{L^{t}\left(\mathbf{T}^{d}\right)},
\end{gathered}
$$

where $\varepsilon=C \rho^{-1+(d-1) / p}$ tends to zero when $\rho$ goes to infinity. This implies that for large $\rho$ the equation

$$
H(k, \mathbf{a}, V) u=H(k, \mathbf{a}, 0) u+V u=0
$$

has no non-zero solutions on the torus, which concludes the proof of the theorem.

As we remarked before, it is possible to weaken the conditions on the electric potential $V$ to those as in [12. This, however, requires a more delicate analytic technique rather than usage of (36). This method is described in [12, and we will not present it here.

\section{OTHER TYPES OF OPERATORS}

In this section we will show that all the types of periodic operators for which absolute continuity has been proven, in spite of their seemingly different appearance, can be reduced to the form (111). Thus, Theorem[7applies. Speaking of the types of operators, we neglect the question about the optimal conditions on the coefficients. In some of the results that we quote these conditions are more relaxed than ours. We did not pursue the goal of obtaining optimal classes of coefficients for which our methods and results would hold.

The following remark is important. With the exception of the trivial case of an isotropic operator (7), the only situations when the absolute continuity has been proven for an operator with variable coefficients in the leading terms are the ones considered in [47] and [48. There the result was established for the Schrödinger operator with variable periodic anisotropic metric in $2 D$ and for the Maxwell operator in a periodic isotropic medium. Although these operators appear to have truly variable coefficients in the leading terms, it will be shown below that in fact they also boil down to (11). In other words, there has not been any true success 
achieved yet for the variable coefficient case. This is not to diminish the role of the remarkable papers [47] and [48], which not only contained important new results, but also introduced some innovative analytic tricks that might be useful in more general situations.

7.1. Schrödinger operator. The standard scalar periodic Schrödinger operator

$$
(D-A)^{2}+V=\sum\left(\frac{1}{i} \frac{\partial}{\partial x_{j}}-a_{j}(x)\right)^{2}+V(x)
$$

is obviously a particular case of (11) for $n=1$. Hence, the results of Theorems 7 and 8 apply.

7.2. Dirac operator. After the absolute continuity of the spectra of the Schrödinger operator with a periodic electric potential was established in 68, the similar result was obtained for the periodic Dirac operator in [16]-[19. This study of the Dirac operator was continued in [9, [13], and 20]-24.

Let us recall what the periodic Dirac operator in $\mathbb{R}^{d}$ is. Let $\alpha_{j}(j=1,2, \ldots, d)$ be Hermitian $m \times m$ matrices for some $m \in \mathbb{N}$ satisfying the relations

$$
\alpha_{j} \alpha_{l}+\alpha_{l} \alpha_{j}=2 \delta_{j l} I
$$

where $\delta_{j l}$ is the Kronecker delta, and $I$ is the unit $m \times m$ matrix. Consider the operator

$$
\mathcal{D}=\frac{1}{i} \sum_{j=1}^{d} \frac{\partial}{\partial x_{j}} \alpha_{j}+V(x)
$$

acting on vector-functions from $\mathbb{R}^{d}$ to $\mathbb{C}^{m}$. Here $V(x)$ is a function with values in $m \times m$ matrices periodic with respect to a lattice $\Gamma$ in $\mathbb{R}^{d}$. Let us assume that $V(x)$ belongs to $L_{r, l o c}$ with $r>2$ when $d=2$, and $V$ is continuous in higher dimensions. If additionally $V$ is self-adjoint, then the operator $\mathcal{D}$ is self-adjoint in $L_{2}\left(\mathbb{R}^{d}\right)$ with the domain $H^{1}\left(\mathbb{R}^{d}\right)$.

Conjecture 14. The periodic self-adjoint Dirac operator $\mathcal{D}$ has absolutely continuous spectrum.

This conjecture has not been proven in full generality even for smooth matrix potentials $V$. As it was explained before, in order to establish absolute continuity, it is sufficient to prove absence of eigenvalues. This must hold also in the nonselfadjoint case.

Conjecture 15. Without the assumption of self-adjointness of the potential $V$ the periodic Dirac operator still has no $L_{2}$-eigenfunctions.

We will now explain under what condition one can reduce study of this problem to the one for an operator (11) and hence prove absolute continuity. We will show then that all cases when the conjecture has been proven fall into this category. We will do this first without paying attention to the conditions on the potential. At this stage the reader may take $V$ to be of the class $C^{1}$. The requirements on $V$ will be specified later.

Assume that there exists an $L_{2}$-eigenfunction $u$ of the Dirac operator, i.e.

$$
\mathcal{D} u=\frac{1}{i} \sum_{j=1}^{d} \frac{\partial u}{\partial x_{j}} \alpha_{j}+V u=\lambda u .
$$


Ellipticity enables is to conclude that then $u \in H^{2}$. This leads to the equality

$$
\mathcal{D}^{2} u=\lambda^{2} u,
$$

or

$$
-\Delta u-i \sum_{j}\left(V(x) \alpha_{j}+\alpha_{j} V(x)\right) \frac{\partial u}{\partial x_{j}}+\left(V^{2}-i \sum_{j} \alpha_{j} \frac{\partial V}{\partial x_{j}}\right) u=\lambda^{2} u .
$$

It is clear that we are in the situation of an operator (11). However, Theorems 5 and77require the "magnetic potential" matrices $a_{j}(x)=V(x) \alpha_{j}+\alpha_{j} V(x)$ to satisfy (13). This leads, according to Theorems 7 and 8 to the following statement:

Theorem 16. Assume that:

1. The average values of the matrix functions $a_{j}(x)=V(x) \alpha_{j}+\alpha_{j} V(x), j=$ $1, \ldots, d$, are equal to zero.

2. The matrices $V(x) \alpha_{j}+\alpha_{j} V(x)$ for different values of $x$ and $j$ satisfy the commutation relation (13).

3. $\left(V(x) \alpha_{j}+\alpha_{j} V(x)\right) \in H_{l o c}^{s}\left(\mathbb{R}^{d}\right)$ with $s>\frac{3}{2} d-1$.

4. $\left(V^{2}-\sum_{j} \alpha_{j} \frac{\partial V}{\partial x_{j}}\right) \in L_{p, l o c}\left(\mathbb{R}^{d}\right)$ with $p=2$ for $d=2$ and $p>d-1$ for $d>2$.

Then the operator (38) has no $L_{2}$-eigenfunctions. In particular, in the selfadjoint case (i.e., when the matrix $V(x)$ is self-adjoint) the spectrum is absolutely continuous.

The main (and probably unnecessary) restriction is the commutation condition (13) on the matrices $\left(V(x) \alpha_{j}+\alpha_{j} V(x)\right)$. We want to point out, however, that in all cases known to the authors when absolute continuity of the periodic Dirac operator has been proven so far, this condition is satisfied. Indeed, [9], 13, and [16-24] assume a very specific types of the potentials. Namely, it is assumed that the operator has the following form:

$$
\sum_{j=1}^{d}\left(\frac{1}{i} \frac{\partial}{\partial x_{j}}-c_{j}\right) \alpha_{j}+V_{0} \alpha_{d+1}+V_{1} I
$$

where $c_{j}, V_{0}$, and $V_{1}$ are scalar functions, and the family $\left\{\alpha_{1}, \ldots, \alpha_{d+1}\right\}$ satisfies the same anti-commutativity condition (37) that has been assumed so far for $\left\{\alpha_{1}, \ldots, \alpha_{d}\right\}$. In this case

$$
V(x)=-\sum_{j=1}^{d} c_{j} \alpha_{j}+V_{0} \alpha_{d+1}+V_{1} I,
$$

and hence for $l \leq d$

$$
\begin{gathered}
2 a_{l}(x)=\left(V(x) \alpha_{l}+\alpha_{l} V(x)\right) \\
=-\sum_{j=1}^{d} c_{j}(x) \alpha_{j} \alpha_{l}+V_{0}(x) \alpha_{d+1} \alpha_{l}+V_{1}(x) \alpha_{l}-\sum_{j=1}^{d} c_{j} \alpha_{l} \alpha_{j}+V_{0} \alpha_{l} \alpha_{d+1}+V_{1} \alpha_{l} \\
=-2 c_{l}(x) I+2 V_{1}(x) \alpha_{l} .
\end{gathered}
$$


Then

$$
\left[a_{l}(x), a_{j}(y)\right]+\left[a_{j}(x), a_{l}(y)\right]=V_{1}(x) V_{1}(y)\left(\left[\alpha_{l}, \alpha_{j}\right]+\left[\alpha_{j}, \alpha_{l}\right]\right)=0,
$$

so the condition (13) is satisfied.

In some cases [24] potentials slightly more general than (39) are considered:

$$
V(x)=-\sum_{j=1}^{d} c_{j} \alpha_{j}+V_{0}+V_{1},
$$

where the matrix function $V_{0}$ commutes and $V_{1}$ anti-commutes with the matrices $\alpha_{1}, \ldots, \alpha_{d}$. One can check, as in the calculation above, that (13) still holds.

\subsection{D operators with variable metric and global isothermal coordinates.}

Most of the known results on absolute continuity of periodic elliptic operators deal with operators with constant coefficients in the principal part (modulo the isotropic operator (7), where the metric conformal to the flat one is eliminated by the substitution (88)). The only exceptions were the innovative papers [47] and [48] by A. Morame. We will consider the operators treated in these papers in this and the next subsections in order to show that they in fact boil down to a Schrödinger operator (11), and hence belong to the realm of operators with constant coefficient leading terms.

The paper 47] contains the proof of absolute continuity of the spectrum of the periodic Schrödinger operator with variable metric in $2 D$ :

$$
\sum_{1 \leq j, k \leq 2}|g|^{-1 / 2}\left(D_{x_{j}}-a_{j}(x)\right) g^{j, k}(x)|g|^{1 / 2}\left(D_{x_{k}}-a_{k}(x)\right)+V(x), \quad x \in \mathbb{R}^{2} .
$$

Here the metric $g(x)$ (a positive definite matrix function) is assumed to be periodic with respect to a lattice $\Gamma$, and of class $C^{\infty}$. The magnetic potential $a$ is $\Gamma$-periodic and belongs to $C^{\infty}$, and the electric potential $V$ is $\Gamma$-periodic and belongs to $L_{\infty}$. The operator, in order to be self-adjoint, needs to be considered in the appropriate $L_{2}$-space, namely in $L_{2}\left(\mathbb{R}^{2} ;|g|^{1 / 2} d x\right)$.

We will show here that even under much milder conditions on the coefficients this operator can be reduced to a scalar periodic Schrödinger operator. Existence of such a reduction does not diminish the relevance of the result and its proof provided in [47. Besides being the first paper where the absolute continuity problem in the presence of variable coefficient second order terms was successfully treated, 47] also contains new ingenious analytic tricks that might be useful in other cases.

Consider the following more general second order periodic operator in $2 D$ :

$$
\begin{gathered}
H=D^{*} g(x) D+a(x) \cdot \nabla+V(x) \\
=-\sum_{1 \leq j, k \leq 2} \frac{\partial}{\partial x_{j}} g_{j, k}(x) \frac{\partial}{\partial x_{k}}+\sum_{1 \leq j \leq 2} a_{j}(x) \frac{\partial}{\partial x_{j}}+V(x),
\end{gathered}
$$

where we do not yet specify conditions on the coefficients $g$, $a$, and $V$ except that $g$ is a positive matrix function, and all the coefficients are periodic with respect to a lattice $\Gamma$ in $\mathbb{R}^{2}$. First let $g(x)$ be a scalar, i.e. $g(x)=b(x) I_{2}$, where $b(x)$ is a sufficiently smooth positive scalar function and $I_{2}$ is the $2 \times 2$ unit matrix. We call such an operator isotropic. Then, as we explained in the introduction, the problem of absolute continuity of the spectrum of such an operator can be transformed to the same problem for the Schrödinger operator $b^{-1 / 2} H \circ b^{-1 / 2}$. Our goal in this section 
is to show that in $2 D$ one can always transform the operator $H$ to an isotropic one by a periodic change of coordinates, and hence reduce the absolute continuity problem to the well-studied Schrödinger case.

First of all, the reader is probably aware of the existence of local coordinate changes that do this. Such coordinates are called isothermal or conformal, and are used for the local study of surfaces in $\mathbb{R}^{3}$ (e.g., Section 13 of Ch. 2 in [25] and p. 376 in [67]), for reduction of second order elliptic PDEs in two variables to the canonical form ([15]), and in complex analysis ([2], 69]). While 25] and 67] assume (for simplicity) significant smoothness (and even analyticity) of the metric $g$, one can find in [2], 15, and [69] mild conditions under which such coordinates exist.

Lemma 17 ( 8 in Ch. IV of [15, and [69]). Let $m$ be a non-negative integer and $\alpha \in(0,1)$. Assume that the entries of the matrix function $g(x)$ in $(40)$ belong to $C^{m+\alpha}\left(\mathbb{R}^{2}\right)$. Then in a neighborhood of any point $x_{0} \in \mathbb{R}^{2}$ there exists a change of variables $y(x) \in C^{m+1+\alpha}\left(\mathbb{R}^{2}\right)$ that transforms the operator (40) to one with the leading term

$$
-\nabla \cdot \sigma(y) \nabla
$$

with a scalar function $\sigma$ that belongs to $C^{m+\alpha}$ in a neighborhood of $y\left(x_{0}\right)$.

So, locally the anisotropy of the operator can be eliminated by switching to the isothermal variables $y$. The interesting observation is that such variables can be chosen globally for a periodic operator. Before going into the details, we want to mention that it was pointed to the first author by L. Friedlander and S. Novikov that the uniformization theorem for Riemann surfaces (see for instance Section 11G in Ch. III of [3]) implies the existence of global periodic isothermal coordinates that do the reduction. Namely, the following statement holds:

Proposition 18. Under the same conditions on the matrix $g(x)$ as in Lemma 17, there exists a global change of variables $\Psi: \mathbb{R}^{2} \rightarrow \mathbb{R}^{2}$ that transforms the operator into an isotropic one and is such that $\Psi(0)=0$ and for any integer vector $\mathbf{n}=$ $\left(n_{1}, n_{2}\right) \in \mathbb{Z}^{2}$

$$
\Psi(x+\mathbf{n})=\Psi(x)+n_{1} \Psi((1,0))+n_{2} \Psi((0,1)) .
$$

The smoothness of the global change $\Psi$ is the same as that of the local one.

The equality (41) means that $\Psi$ transforms the original $\mathbb{Z}^{2}$-periodic operator (40) into an isotropic one which is periodic with respect to the lattice generated by the basis vectors $\Psi((1,0))$ and $\Psi((0,1))$. In particular, the operator is transformed into one for which the absolute continuity problem can be resolved by reduction to the Schrödinger case. This implies the result of [47] under much milder conditions on the coefficients than in [47] (see for instance the discussion in [14]).

Proof of Proposition 18. The local isothermal coordinates that exist according to Lemma 17 can be chosen to preserve orientation. They provide an atlas of local coordinate charts on the torus $\mathbb{T}^{2}=\mathbb{R}^{2} / \mathbb{Z}^{2}$. We can think that they map local coordinate patches onto the unit disk in $\mathbb{C}$ (identified with $\mathbb{R}^{2}$ ). Then transforms between the intersecting local charts are orientation-preserving and conformal, and hence holomorphic. This introduces a structure of a Riemannian surface $\mathcal{R}$ on $\mathbb{T}^{2}$. Since $\mathcal{R}$ is a compact Riemannian surface of genus $g=1$, there exists a unique (up to a constant factor) holomorphic differential (differential 1-form) $\omega$, since the dimension of the vector space of such differentials is equal to the genus 
(e.g., Theorem 10-3 in [62 and Section 24A in Ch. V of [3]). Holomorphy means that in the local isothermal coordinates $x, y$ the differential looks like $f(z) d z$ with a holomorphic function $f(z)$, where $z=x+i y$. This differential has no zeros (i.e., $f(z)$ has no zeros in each local chart), since otherwise we could use shifts to create zeros at an arbitrary point, which would contradict the one-dimensionality of the space of such differentials. Let us consider the covering mapping

$$
\mathbb{R}^{2} \rightarrow \mathbb{R}^{2} / \mathbb{Z}^{2}=\mathcal{R}
$$

and pull back this differential $\omega$ to a differential $\Omega$ on the whole $\mathbb{R}^{2}$. Let us also fix a point (for instance, the origin 0 ) in $\mathbb{R}^{2}$. Then, integrating $\Omega$ from this point to a variable point $x$ in the plane, we get the mapping

$$
\Psi(x)=\int_{0}^{x} \Omega
$$

from $\mathbb{R}^{2}$ into the complex plane. It is easy to see that this mapping is a homeomorphism of $\mathbb{R}^{2}$. It maps the lattice of periods isomorphically onto another lattice and gives the global periodic isothermal coordinates. Notice that due to analyticity of the differential there was no loss of smoothness in the process of moving from local to global isothermal coordinates. This finishes the proof.

7.4. Maxwell operator. Consider a $3 D$ medium described by positive electric permittivity $\varepsilon(x)$ and magnetic permeability $\mu(x)$. Both functions are scalar (thus the medium is isotropic), periodic with respect to a lattice $\Gamma$ in $\mathbb{R}^{3}$, measurable, and bounded away from zero and infinity:

$$
0<c<\varepsilon(x), \quad \mu(x)<C<\infty .
$$

Later on in this section it will be assumed that these functions are of the class $C^{1}$. We will denote by $\mathbf{E}(x)$ and $\mathbf{H}(x)$ the electric and magnetic fields. Consider the Hilbert space $\mathcal{L}=L_{2}\left(\mathbb{R}^{3} ; \mathbb{C}^{3}, \varepsilon\right) \oplus L_{2}\left(\mathbb{R}^{3} ; \mathbb{C}^{3}, \mu\right)$ of pairs $(\mathbf{E}, \mathbf{H})$. Here $L_{2}\left(\mathbb{R}^{3} ; \mathbb{C}^{3}, s\right)$ denotes the $L_{2}$-space with respect to the measure $s d x$ of vector functions on $\mathbb{R}^{3}$ with values in $\mathbb{C}^{3}$. The subspace $L \subset \mathcal{L}$ consists of pairs $(\mathbf{E}, \mathbf{H})$ that satisfy the zero divergence conditions

$$
\nabla \cdot \mu \mathbf{H}=\mathbf{0}, \quad \nabla \cdot \varepsilon \mathbf{E}=\mathbf{0} .
$$

The Maxwell operator $M$ acts in $L$ as follows:

$$
M\left(\begin{array}{l}
\mathbf{E} \\
\mathbf{H}
\end{array}\right)=\left(\begin{array}{ll}
0 & i \frac{1}{\varepsilon} \nabla \times \mathbf{H} \\
-i \frac{1}{\mu} \nabla \times \mathbf{E} & 0
\end{array}\right),
$$

with the domain consisting of fields $(\mathbf{E}, \mathbf{H})$ such that $\nabla \times \mathbf{E}$ and $\nabla \times \mathbf{H}$ belong to $L_{2}\left(\mathbb{R}^{3} ; \mathbb{C}^{3}\right)$. This operator is self-adjoint $[7$. The reader can find the spectral theory of Maxwell operators developed in high generality in [7].

It is believed that the spectrum of the Maxwell operator $M$ in a periodic medium is absolutely continuous. This question is relevant, for instance, for the theory of photonic crystals [38. This belief was justified for the case of $C^{\infty}$ functions $\varepsilon$ and $\mu$ in [48]. The goal of this section is to show that the essence of what was done in [48] boils down to a reduction of the problem to a non-self-adjoint matrix Schrödinger operator of the type (11), so that Theorem $[7$ applies. This was noticed by T. Suslina [63]. 
The crucial observation of the paper [48] was the following statement, which can be verified by a direct calculation:

Lemma 19. Let the functions $\varepsilon$ and $\mu$ be of class $C^{2}$, and let $\mathbf{u} \in H_{\text {loc }}^{2}\left(\mathbb{R}^{3}, \mathbb{C}^{3}\right)$ be such that $\nabla \cdot \varepsilon \mathbf{u}=\mathbf{0}$. Then

$$
\nabla \times \frac{1}{\mu} \nabla \times \mathbf{u}=-\frac{1}{\mu \sqrt{\varepsilon}} \Delta(\sqrt{\varepsilon} \mathbf{u})+\left(\varepsilon \nabla\left(\frac{1}{\mu \varepsilon}\right)\right) \times(\nabla \times \mathbf{u})+W \mathbf{u},
$$

where $W$ is a $3 \times 3$ matrix whose entries are expressible in terms of $\varepsilon$ and $\mu$, and the equality holds in the distributional sense.

Suppose now that we have an $L_{2}$-eigenfunction $(\mathbf{E}, \mathbf{H})$ of the Maxwell operator. This means that

$$
\left\{\begin{array}{l}
i \frac{1}{\varepsilon} \nabla \times \mathbf{H}=\lambda \mathbf{E} \\
-i \frac{1}{\mu} \nabla \times \mathbf{E}=\lambda \mathbf{H}
\end{array}\right.
$$

In this case further application of the curl operator is possible, which leads to

$$
\left\{\begin{array}{l}
\nabla \times \frac{1}{\mu} \nabla \times \mathbf{E}=i \lambda \nabla \times \mathbf{H}=\lambda \varepsilon \mathbf{E} \\
\nabla \times \frac{1}{\varepsilon} \nabla \times \mathbf{H}=-i \lambda \nabla \times \mathbf{E}=-\lambda \mu \mathbf{H} \\
\nabla \cdot \varepsilon \mathbf{E}=\mathbf{0} \\
\nabla \cdot \mu \mathbf{H}=\mathbf{0}
\end{array}\right.
$$

Using the lemma for the first two equations, we now conclude that

$$
\left\{\begin{array}{l}
-\frac{1}{\mu \sqrt{\varepsilon}} \Delta(\sqrt{\varepsilon} \mathbf{E})+\left(\varepsilon \nabla\left(\frac{1}{\mu \varepsilon}\right)\right) \times(\nabla \times \mathbf{E})+W_{1} \mathbf{E}-\lambda \varepsilon \mathbf{E}=0, \\
-\frac{1}{\varepsilon \sqrt{\mu}} \Delta(\sqrt{\mu} \mathbf{H})+\left(\mu \nabla\left(\frac{1}{\mu \varepsilon}\right)\right) \times(\nabla \times \mathbf{H})+W_{2} \mathbf{H}+\lambda \mu \mathbf{H}=0,
\end{array}\right.
$$

where $W_{j}$ are $3 \times 3$ matrices. Using the equations (45) again to eliminate the curl terms, we obtain the system

$$
\left\{\begin{array}{l}
-\frac{1}{\mu \sqrt{\varepsilon}} \Delta(\sqrt{\varepsilon} \mathbf{E})+i \lambda \mu\left(\varepsilon \nabla\left(\frac{1}{\mu \varepsilon}\right)\right) \times \mathbf{H}+W_{1} \mathbf{E}-\lambda \varepsilon \mathbf{E}=0, \\
-\frac{1}{\varepsilon \sqrt{\mu}} \Delta(\sqrt{\mu} \mathbf{H})-i \lambda \varepsilon\left(\mu \nabla\left(\frac{1}{\mu \varepsilon}\right)\right) \times \mathbf{E}+W_{2} \mathbf{H}+\lambda \mu \mathbf{H}=0 .
\end{array}\right.
$$

Let us introduce a new six-dimensional vector-function $\mathbf{v}=(\sqrt{\varepsilon} \mathbf{E}, \sqrt{\mu} \mathbf{H})$. It belongs to $L_{2}$, due to the assumptions that $(\mathbf{E}, \mathbf{H})$ is in $L_{2}$ and the functions $\varepsilon$ and $\mu$ are bounded. For this vector-function we rewrite (47) as the equation

$$
-\Delta \mathbf{v}+V \mathbf{v}=0
$$

where $V$ is a $6 \times 6$ periodic matrix-function with bounded entries obtained from $\varepsilon$, $\mu$, and $\lambda$ according to the above procedure. This equation is an eigenvalue problem for the matrix operator $-\Delta+V$, which is obviously of the type (11). This completes our reduction. Notice that the matrix $V$ is in general not self-adjoint, despite the fact that we started with a self-adjoint operator $\dot{M}$. According to Theorem 7 , this is irrelevant, and (48) has no non-zero $L_{2}$-solutions.

Theorem 20. Under the conditions stated in this section, the Maxwell operator $M$ has absolutely continuous spectrum. 


\section{CONCluding REMARKS}

1. It remains a challenge to prove the absolute continuity of the spectrum of the general second order periodic elliptic operator

$$
-\sum_{1 \leq j, k \leq 2} \frac{\partial}{\partial x_{j}} g_{j, k}(x) \frac{\partial}{\partial x_{k}}+\sum_{1 \leq j \leq 2} a_{j}(x) \frac{\partial}{\partial x_{j}}+V(x)
$$

in dimensions higher than two, even for smooth metric $\left\{g_{j, k}(x)\right\}$. The trick with isothermal coordinates works only in $2 D$. Similarly, absolute continuity has not been proven for the periodic Maxwell operator in anisotropic media, i.e. when the material tensors $\varepsilon(x)$ and $\mu(x)$ are matrices rather than scalars. One can assume arbitrary smoothness of $\varepsilon$ and $\mu$. It looks like new approaches are needed in order to treat these problems. One of them could be to obtain appropriate theorems on the allowed rate of decay of solutions that generalize those of [30] and [46. If one could prohibit super-exponential decay of eigenfunctions, then results of [36, 37] would imply the absolute continuity (see the corresponding discussion in the Introduction). The other obvious possibility is to try to push through the Thomas approach. This, however, might be too difficult in the presence of variable coefficients in the leading terms. As this paper makes clear, all known results essentially deal with constant coefficient principal part only.

2. In the previous remark we listed the problems that remain unresolved in the case of smooth coefficients. For some other operators, sufficiently smooth coefficients do not pose a challenge anymore, but reducing conditions on the coefficients is an important task. The optimal conditions on the electric potential in terms of standard function spaces have already been approached in [12, 63], and [55]-[58] (see also [14] and [33], where the potential is allowed to contain parts supported on a graph, or delta-type terms). This is not so for the magnetic potential. Conditions imposed in [65] and in the current paper are definitely unnecessarily strong. One can find in [12] a reasonable conjecture about the optimal conditions on $a$, which has been justified for $d=2$, and some recent advances in [24]. The situation is even worse with the coefficients in the leading terms. Namely, the reduction (8) of the isotropic operator (7) to the Schrödinger case works only when the metric $\rho(x)$ is sufficiently smooth (for instance, belongs to $H_{l o c}^{2}$ ). This, however, is far from what we need for applied problems, since many applications work with composite media, where the coefficient $\rho(x)$ is piecewise constant. The same remark applies to the discussion in Section 7.3 where isothermal coordinates are used. Analogously, applications to photonic crystals ([32, [38]) require considering piecewise constant dielectric permittivity $\varepsilon(x)$ and magnetic permeability $\mu(x)$. There is little doubt that the spectra must be absolutely continuous in these cases, but proving this might require new techniques.

3. We are tempted to say that the commutation condition (13) looks irrelevant and should not be necessary. There are only two things that stop us from doing so. First of all, as it is shown above, all known results relate to the "commutative" situation only. Second, the simplest "non-commutative" case of the Dirac operator with arbitrary smooth matrix potential $V$ has not been covered, in spite of the significant attention paid to the Dirac operator. So, one cannot exclude the possibility of surprises. Let us now take a closer 
look at condition (13). In its absence the proof stalls at the inversion of the model operator $\frac{\partial}{\partial \bar{z}}+F(z)$ on the torus, when the matrix function $F(z)$ is not commutative. In order for the process of gauging away most of the magnetic potential to go through, one needs to find an invertible matrix function $B(z)$ (the replacement of the exponent $\exp i \phi(z)$ that was used before) on the torus $\mathbb{T}^{2}=\mathbb{R}^{2} / \mathbb{Z}^{2}$ such that

$$
\frac{\partial B}{\partial \bar{z}}=B F
$$

This problem is known in complex analysis in several variables. It is known (see, for instance [45]) that its solution always exists locally. It is straightforward to check that the ratios of local solutions are holomorphic. This means that the local solutions determine an analytic vector bundle on the torus $\mathbb{T}^{2}$. Thus, existence of the needed global solution is equivalent to analytic triviality of this bundle. One wanders whether a version of Sobolev's trick (65. and Section 5.3.4 of the current paper) could let one eliminate this analytic obstruction and gauge away most of the magnetic potential. In the commutative case this is not hard to do, since one can describe the obstacle as non-vanishing of some Fourier coefficients of $F$; but this is probably impossible in general.

After the paper was submitted to the editors, the preprint "On the spectrum of a class of second order periodic elliptic differential operators" by L. Friedlander appeared that contained a major breakthrough for the case of variable coefficients in the leading terms of the operator.

\section{ACKNOWLEDGMENTS}

The authors express their gratitude to Professors M. Birman, L. Danilov, L. Friedlander, R. Novikov, S. Novikov, A. Sobolev, Z. Shen, Z. Sun, T. Suslina, A. Tumanov, A. Pankov, and V. Palamodov for information and comments. We are thankful to Professor T. Suslina for many important remarks about the first version of the manuscript.

This research was partly sponsored by the NRC through a COBASE Grant, NSF through the Grants DMS 9610444 and DMS 0072248, and by the Department of the Army, Army Research Office, and the state of Kansas through a DEPSCoR Grant. The authors thank the NRC, NSF, ARO, and the state of Kansas for this support. The content of this paper does not necessarily reflect the position or the policy of the federal government, and no official endorsement should be inferred.

\section{REFERENCES}

1. M.S. Agranovich and M.I. Vishik, Elliptic problems with a parameter and parabolic problems of general type, Russian Math. Surveys 19 (1964), no.3, 53-157. MR 33:415

2. L. V. Ahlfors, Lectures on Quasiconformal Mappings, Van Nostrand Mathematical Studies, No. 10, Van Nostrand, New York, 1966. MR 34:336

3. L. V. Ahlfors and L. Sario, Riemann Surfaces, Princeton Univ. Press, Princeton NJ 1960. MR 22:5729

4. N.W. Ashcroft and N.D. Mermin, Solid State Physics, Holt, Rinehart and Winston, New York-London 1976.

5. W. Axmann, P. Kuchment, and L. Kunyansky, Asymptotic methods for thin high contrast $2 D$ PBG materials, Journal of Lightwave Technology, 17 (1999), no.11, 1996-2007.

6. A. Berthier, On the point spectrum of Schrödinger operators, Ann. Scient. Ec. Norm. Super., 4 serie, 15 (1982), 1-15. MR 84i:35106 
7. M. Birman and M. Solomyak, $L_{2}$-theory of the Maxwell operator in arbitrary domains, Russian Math. Surveys 42 (1987), no 6: 75-96. MR 89e:35127]

8. M. Sh. Birman and T. A. Suslina, The two-dimensional periodic magnetic Hamiltonian is absolutely continuous. (Russian) Algebra i Analiz 9 (1997), no. 1, 32-48; translation in St. Petersburg Math. J. 9 (1998), no. 1, 21-32. MR 98g:47038

9. M. Sh. Birman and T. A. Suslina, The periodic Dirac operator is absolutely continuous, Integr. Equat. and Operator Theory 34 (1999), 377-395. MR 2000h:47068

10. M. Sh. Birman and T. A. Suslina, Absolute continuity of the two-dimensional periodic magnetic Hamiltonian with discontinuous vector-valued potential. (Russian) Algebra i Analiz 10 (1998), no. 4, 1-36; translation in St. Petersburg Math. J. 10 (1999), no. 4, 579-601. MR 99k:81060

11. M. Sh. Birman and T. A. Suslina, Two-dimensional periodic Pauli operator. The effective masses at the lower edge of the spectrum, in Mathematical Results in Quantum Mechanics (QMath7, Prague, June 22-26,1998), J. Dittrich, P. Exner, et al (Editors), 13-31, Operator Theory, Adv. and Appl., v. 108, Birkhäuser, Basel, 1999. MR 2000g:81049

12. M. Sh. Birman and T. A. Suslina, Periodic magnetic Hamiltonian with a variable metric. The problem of absolute continuity, Algebra i Analiz 11 (1999), no.2. English translation in St. Petersburg Math J. 11 (2000), no.2, 203-232. MR 2000i:35026

13. M. Sh. Birman and T. A. Suslina, On the absolute continuity of the periodic Schrödinger and Dirac operators with magnetic potential, in Differential Equations and Mathematical Physics (Birmingham, AL, 1999), AMSIP Stud. Adv. Math., vol. 16, Amer. Math. Soc., Providence, RI, 2000, pp. 41-49. MR 2000m:00026

14. M. Sh. Birman, T. A. Suslina, and R. G. Shterenberg, Absolute continuity of the spectrum of a two-dimensional Schrödinger operator with potential supported on a periodic system of curves, Preprint ESI no. 934, http://www.esi.ac.at, 2000.

15. R. Courant and D. Hilbert, Methods of Mathematical Physics, Vol. II. Partial differential equations. John Wiley \& Sons, Inc., New York, 1989. MR 90k:35001

16. L. Danilov, Zone structure of the spectrum of the Dirac operator with periodic potential, in: Nonlinear Oscillations and Control Theory, Ustinov 1985, 83-90. (Russian)

17. L. Danilov, On the spectrum of the Dirac operator with periodic potential, Acad. Sci. USSR, Ural Branch, preprint, Sverdlovsk, 1987. (Russian)

18. L. Danilov, On the spectrum of the Dirac operator in $\mathbb{R}^{\mathbf{n}}$ with periodic potential. (Russian) Teoret. Mat. Fiz. 85 (1990), no. 1,41-53; translation in Theoret. and Math. Phys. 85 (1990), no. 1, 1039-1048 (1991). MR 92a:35119

19. L. Danilov, The spectrum of the Dirac operator with periodic potential I, preprint, no.4588B91, VINITI, Moscow 1991, 36 pp. (Russian)

20. L. Danilov, Resolvent estimates and the spectrum of the Dirac operator with a periodic potential. (Russian) Teoret. Mat. Fiz. 103 (1995), no. 1, 3-22; translation in Theoret. and Math. Phys. 103 (1995), no. 1, 349-365. MR 98f:35112

21. L. Danilov, On the spectrum of the two-dimensional periodic Dirac operator. (Russian) Teoret. Mat. Fiz. 118 (1999), no. 1, 3-14; translation in Theoret. and Math. Phys. 118 (1999), no. 1, 1-11. MR 2000h:35117

22. L. Danilov, Absolute continuity of the spectrum of the periodic Dirac operator, Diff. Uravneniya, 36 (2000), no.2, 233-240; English transl., Differential Equations 36 (2000), 262-271. MR 2001f: 42038

23. L. Danilov, On the spectrum of the periodic Dirac operator, Teoret. i Mat. Fiz. 124 (2000), no.1, 3-17; English transl. in Theoret. Math. Phys. 124 (2000). CMP 2001:10

24. L. Danilov, On absolute continuity of spectra of periodic Schrödinger and Dirac operators I, preprint, no. 1683-B00, VINITI, Moscow, 2000. (Russian)

25. B. A. Dubrovin, A. T. Fomenko, and S. P. Novikov, Modern Geometry - Methods and Applications, Part I, 2nd ed., Springer Verlag, New York, 1992. MR 92h:53001

26. B. A. Dubrovin and S. P. Novikov, Ground states in a periodic field. Magnetic Bloch functions and vector bundles, Soviet Math. Dokl. 22 (1980), 240-244. MR 82c:81181

27. B. A. Dubrovin and S. P. Novikov, Ground states of a two-dimensional electron in a periodic magnetic field, Soviet Physics JETF 52 (1980), 511-516. MR 82i:81018

28. M. S. P. Eastham, The Spectral Theory of Periodic Differential Equations, Scottish Acad. Press, Edinburgh-London, 1973. 
29. L. Ehrenpreis, Fourier Analysis in Several Complex Variables, Wiley-Interscience, New YorkLondon 1970. MR 44:3066

30. R. Froese, I. Herbst, M. Hoffmann-Ostenhof, and T. Hoffmann-Ostenhof, $L_{2}$-lower bounds to solutions of one-body Schrödinger equations, Proc. Royal Soc. Edinburgh, 95A (1983), 25-38. MR 86a:35044

31. R. Hempel and I. Herbst, Strong magnetic fields, Dirichlet boundaries, and spectral gaps, Comm. Math. Phys. 164 (1995), 237-259. MR 96a:81026

32. J.D. Joannopoulos, R.D. Meade, and J. N. Winn, Photonic Crystals. Molding the Flow of Light, Princeton Univ. Press, Princeton, NJ, 1995.

33. Yu. Karpeshina, Spectrum and eigenfunctions of Schrödinger operator with zero-range potential of the homoeneous lattice type in three dimensional space, Ter. i Mat. Fiz. 57 (1983), no.2, 304-313; Engl. ransl. in Theor. and Math. Phys. 57 (1983), 1156-1162. MR 86b:81016

34. Yu. Karpeshina, Perturbation Theory for the Schrödinger Operator with a Periodic Potential. Lecture Notes in Mathematics, v. 1663. Springer-Verlag, Berlin, 1997. MR 2001i:35002

35. H. Knörrer and E. Trubowitz, A directional compactification of the complex Bloch variety, Comment. Math. Helv. 65 (1990), no. 1, 114-149. MR 91k:58134

36. P. Kuchment, Floquet Theory for Partial Differential Equations, Russian Math. Surveys, 37 (1982), no.4, 1-60. MR 84b:35018

37. P. Kuchment, Floquet Theory for Partial Differential Equations, Birkhäuser Verlag, Basel 1993. MR 94h:35002

38. P. Kuchment, Mathematics of photonic crystals, in Mathematical Modeling in Optical Science, G. Bao, L. Cowsar, and W. Masters (Editors) SIAM, 2001.

39. P. Kuchment and L. Kunyansky, Spectral properties of high-contrast band-gap materials and operators on graphs, Experimental Mathematics 8 (1999), no.1, 1-28. MR 2000c:78027

40. P. Kuchment and L. Kunyansky, Differential operators on graphs and photonic crystals, to appear in Adv. Comp. Math.

41. P. Kuchment and S. Levendorskiî, On the absolute continuity of spectra of periodic elliptic operators, in Mathematical Results in Quantum Mechanics (QMath7, Prague, June 2226,1998), J. Dittrich, P. Exner, et al (Editors), 291-297, Operator Theory, Adv. and Appl., v. 108, Birkhäuser, Basel, 1999. MR 2001c:35166

42. P. Kuchment and Y. Pinchover, Integral representations and Liouville theorems for solutions of periodic elliptic equations, J. Funct. Anal. 181 (2001), 402-446. CMP 2001:10

43. P. Kuchment and B. Vainberg, On embedded eigenvalues of perturbed periodic Schrödinger operators, in Spectral and Scattering Theory (Newark, DE, 1997), 67-75, Plenum, New York, 1998. MR 99e:35032

44. P. Kuchment and B. Vainberg, On absence of embedded eigenvalues for Schrödinger operators with perturbed periodic potentials, Commun. PDE 25 (2000), no. 9-10, 1809-1826. CMP 2000:17

45. B. Malgrange, Lectures on the theory of functions of several complex variables, Tata Institute of Fundamental Research, Bombay, Springer-Verlag, Berlin 1958. MR 85c:32001 (reprint)

46. V. Meshkov, On the possible rate of decay at infinity of solutions of second order partial differential equations, Mat. Sbornik, 182 (1991), no.3, 364-383. English translation in Math. USSR Sbornik 72 (1992), no.2, 343-351. MR 92d:335032

47. A. Morame, Absence of singular spectrum for a perturbation of a two-dimensional LaplaceBeltrami operator with periodic electro-magnetic potential, J. Phys. A: Math. Gen. 31 (1998), 7593-7601. MR 99i:81039

48. A. Morame, The absolute continuity of the spectrum of Maxwell operator in periodic media, J. Math. Phys. 41 (2000), 7099-7108. CMP 2001:01

49. G. Nakamura, Z. Sun, and G. Uhlmann, Global identifiability for an inverse problem for the Schrödinger equation in a magnetic field, Math. Ann. 303 (1995), no. 3, 377-388. MR 96m:35336

50. S.Novikov, Two-dimensional Schrödinger operators in the periodic fields, Itogi Nauki i Tekhn.: Sovremennye Problemy Mat., vol. 23, VINITI, Moscow, 1983, 3-32; English transl., J. Soviet Math. 28 (1985), 1-19. MR 85i:81020

51. V. P. Palamodov, Linear Differential Equations with Constant Coefficients, Springer-Verlag, Berlin 1970. MR 41:8793

52. V. Palamodov, Harmonic synthesis of solutions of elliptic equations with periodic coefficients, Ann. Inst. Fourier 43 (1993). 751-768. MR 95f:35037 
53. A. Plis, Non-uniqueness in Cauchy's problem for differential equations of elliptic type, J. Math. Mech. 9 (1960), 557-562. MR 22:12305

54. M. Reed, B. Simon, Methods of Modern Mathematical Physics, Vol.IV: Analysis of Operators, Academic Press, New York, 1978. MR 58:12429c

55. Z. Shen, On absolute continuity of the periodic Schrödinger operators, Internat. Math. Res. Notices 2001, no. 1, 1-31.

56. Z. Shen, The periodic Schrödinger operator with potentials in the C. Fefferman-Phong class, Preprint \#99-15, Math. Dept., Univ. of Kentucky, 1999 and \#99-455 in the Texas Math Physics archive, 1999 http://www.ma.utexas.edu/mp_arc.

57. Z. Shen, The periodic Schrödinger operator with potentials in the Morrey-Campanato class, Preprint 1999.

58. Z. Shen, Absolute continuity of periodic Schrödinger operators with potentials in the Kato class, Preprint \#00-294 in the Texas Math Physics archive, 2000 http://www.ma.utexas.edu/mp_arc.

59. M. Shubin, Spectral theory and the index of elliptic operators with almost periodic coefficients, Russian Math. Surveys 34 (1979), no. 2, 109-157. MR 81f:35090

60. J. Sjostrand, Microlocal analysis for the periodic magnetic Schrödinger equation and related questions, in : Microlocal Analysis and Applications, Lect. Notes in Math., v.1495, 237-332, Springer-Verlag, Berlin, 1991. MR 94f:35119

61. M.M. Skriganov, Geometric and arithmetic methods in the spectral theory of multidimensional periodic operators, Trudy Mat. Inst. Steklov. 17 (1985); English transl., Proc. Steklov Inst. Math. 1987, no. 2 (171). MR 87h:47110; MR 88g:47038

62. G. Springer, Introduction to Riemann Surfaces, Chelsea, New York 1981. MR 19:1169g (orig. ed.)

63. T. Suslina, Absolute continuity of the spectrum of periodic operators of mathematical physics, Journees Equations aux Derivees Partielles, Nantes, 5-9 Juin 2000, GDR 1151 (CNRS). MR 2001f:35295

64. Z. Sun, An inverse boundary value problem for Schrödinger operators with vector potentials, Trans. Amer. Math. Soc. 338 (1993), no. 2, 953-969. MR 93j:35066

65. A. Sobolev, Absolute continuity of the periodic magnetic Schrödinger operator, Inventiones Mathematicae 137 (1999), 85-112. MR 2000g:35028

66. A. Sobolev and J. Walthoe, Absolute continuity in periodic waveguides, Preprint, September 2000 .

67. M. Taylor, Partial Differential Equations. I. Basic Theory, Applied Mathematical Sciences, v. 115. Springer-Verlag, New York, 1996. MR 98b:35002

68. L. E. Thomas, Time dependent approach to scattering from impurities in a crystal, Comm. Math. Phys. 33 (1973), 335-343. MR 48:13084

69. I. N. Vekua, Generalized Analytic Functions, Pergamon Press, London; Addison Wesley Pub. Co., Inc., Reading, Mass. 1962. MR 27:321

70. M. Zaidenberg, S. Krein, P. Kuchment, and A. Pankov, Banach bundles and linear operators, Russian Math. Surveys 30 (1975), no.5, 115-175. MR 54:3741

71. J. Zak, Magnetic translation group I, II, Phys. Rev. 134 (1964), no. 6A, A1602-A1611. MR 31:2031. MR 31:2032

Department of Mathematics and Statistics, Wichita State University, Wichita, KANSAS 67260-0033

Current address: Department of Mathematics, Texas A\&M University, College Station, Texas 77843-3368

E-mail address: kuchment@math.tamu.edu

Department of Mathematics and Statistics, Wichita State University, Wichita, KANSAS 67260-0033

Current address: Department of Mathematics, Rostov State Academy of Economics, Rostovon-Don, Russia

E-mail address: leven@ns.rnd.runnet.ru 